\title{
L'émergence silencieuse de la complexité en impression 3D
}

\section{The silent emergence of complexity in 3D printing}

\author{
Jean-Claude André ${ }^{1}$ \\ ${ }^{1}$ LRGP UMR 7274 CNRS-UL, 1, rue Grandville - F54000 Nancy
}

RÉSUMÉ. Inventé en 1984 en quelques jours, le concept de fabrication additive s'est développé assez rapidement pour atteindre un marché de l'ordre de 40 Milliards $€ /$ an avec un taux d'augmentation annuel de $20 \%$. Si la première technologie a été la polymérisation résolue dans l'espace de résines induite par de la lumière, d'autres modes de fabrication ont vu le jour depuis cette date fondatrice permettant la réalisation d'objets dans de multiples matières. A partir des premières preuves de concept, des améliorations incrémentales mono-disciplinaires ont été menées conduisant au succès actuel.

Aujourd'hui d'autres espaces applicatifs se dessinent en exploitant l'aptitude de certains matériaux à changer de forme (et de fonctionnalité) en présence d'une stimulation. C'est ainsi que sont nées deux technologies sœurs, l'impression 4D et le bio-printing avec des marchés potentiels de l'ordre de plusieurs milliers de milliards $€ /$ an. Mais, pour que ces transitions aient le succès espéré, il faut accepter d'explorer le compliqué (4D) et le complexe (Bioprinting).

Ce constat impose donc une réflexion épistémologique à laquelle les scientifiques doivent impérativement s'intéresser pour tenter de trouver des voies de progrès si l'on veut éviter de se cantonner dans son " quant-à-soi » avec une accumulation de preuves de concepts.

Le présent article fait des rappels sur le thème de la fabrication additive, mais surtout tente de montrer comment la complexité s'est introduite silencieusement dans la dynamique de recherche sur le thème, elle-même limitée par une faiblesse numérique de chercheurs et par la difficulté ordinaire des traitements de problèmes inverses et des approches téléologiques en recherche.

ABSTRACT. Invented in 1984 in a few days, the concept of additive manufacturing has been developed quickly enough to reach a global market of around 40 billion $€ /$ year with a $20 \%$ annual growth rate. Although the first technology was the space resolved polymerization of resins induced by light, other manufacturing methods have emerged since this founding date allowing the realization of objects in multiple materials. From the first proofs of concept, incremental mono-disciplinary improvements have been made leading to the current success.

Today, other application spaces are emerging by exploiting the ability of certain materials to change their shape (and functionality) in the presence of a stimulation. This is how two sister technologies, 4D printing and bio-printing, were born with potential markets of several trillion $€ /$ year. But, for these transitions to be as successful as expected, we must accept to explore complication (4D) and complexity (Bio-printing).

This observation therefore imposes an epistemological reflection into which scientists should deeply involve, in order to find ways of progress that avoid confining ourselves to an "autistic conduct" with an accumulation of proof of concepts.

This article provides reminders on the theme of additive manufacturing, but above all it attempts to show how complexity has silently entered the dynamics of research on the theme, itself limited by the small number of researchers and by the classical difficulty of treating inverse problems and teleological approaches in research.

MOTS-CLÉS. Fabrication additive, impression 3D volumique, impression 4D, bio-printing, rupture technologique, attractivité, complexité, interdisciplinarité, convergence.

KEYWORDS. Additive manufacturing, 3D volume printing, 4D printing, bio-printing, technological breakthrough, attractiveness, complexity, interdisciplinarity, convergence. 
«Il existe aussi des brouillards artificiels que nous prenons pour des remparts ; ils constituent les principaux obstacles, car ils bloquent à la racine même tout processus d'imagination alternative » (Viveret, 2012).

«Chaque progrès de vitesse est célébré par les médias comme un succès et adopté comme tel par le public. Mais l'expérience montre que plus nous gagnons du temps, moins nous en avons. Plus nous allons vite, plus nous sommes harcelés » (Ellul, 1988).

«L'imagination des chercheurs couplée au capitalisme intelligent n'a guère de limites dès lors qu'il s'agit de fantasmer un monde allégé nous libérant du maximum technologique possible des contraintes matérielles et corporelles qui nous enchaînent au monde. Il sera facile toutefois de montrer que cette libération se retourne dialectiquement en asservissement » (Hunyadi, 2015).

«Le progrès, plus qu'une figure ardente, c'est cette fièvre inquiète, ce continuel transport qui travaille la société toute entière, qui ne lui laisse ni trêve, ni repos ni bonheur. Quel traitement il faut à ce mal, on l'ignore » (Töpffer, 1835).

\section{Introduction : A l'origine de l'impression 3D}

De façon très réductrice probablement, un chercheur en sciences pour l'ingénieur utilise des savoirs scientifiques - ce qui est acquis et reconnu - des savoirs pratiques et sa possible intuition pour créer des connaissances nouvelles. La formation initiale des personnels de la recherche est en général purement scientifique, associée à une soumission sans critique aux dogmes ou aux paradigmes disciplinaires, car il faut bien, par conditionnement, réussir aux examens... Alors, comment passe-t-on :

- Du su à l'imaginé ?

- Du conforme à la divergence créative, puis à l'innovation ?

- De la formation rigide, mais efficiente, à une dynamique de recherche et/ou à des transgressions reliées aux innovations?

Les pédagogues nous ont façonné/formaté par les programmes scolaires inscrits dans le système de valeurs du moment pour devenir à notre tour détenteurs de savoirs. Ceci est à l'image de nos maîtres, prêtres dépositaires d'une connaissance, généralement scientifique, établie, rassurante et donc juste. En effet, la société a besoin d'acteurs maîtrisant bien les connaissances scientifiques et techniques dures pour maintenir l'outil de production, voire le faire progresser sur des bases incrémentales. Le domaine scientifique des interactions lumière-matière abordé par l'auteur est à la fois une science d'approfondissement et une science de transfert, où il est possible d'agir de manière flexible, pour promouvoir des actions aux interfaces. Celles-ci permettent d'aller au-delà de la remarque de François Kourilsky, ancien Directeur Général du CNRS, écrite en 2002 : «Combien de chercheurs poussent la spécialisation jusqu'au désintérêt à franchir les frontières de leur discipline. Pire, certains ne s'intéressent même plus aux zones frontières de la discipline elle-même » (André, 2016).

Dans le domaine d'interfaces disciplinaires impliquant le photon, nombre de déplacements sont possibles dans un espace allant de la physique (physique des liquides), la chimie-physique (cinétiques photo-physiques et photochimiques, spectroscopies), le génie des procédés photochimiques, à l'instrumentation optique, aux capteurs optiques, à l'interface optique-biologie pour atteindre provisoirement la stéréo-lithographie laser (le premier procédé de fabrication additive) et aujourd'hui les nano-médicaments anti-cancers stimulés par de la lumière, les capes d'invisibilité, etc. En ce sens l'activité a-t-elle relevé de la «vraie » science parce qu'acceptée par les 
pairs dans des revues à comité de lecture et par quelques dizaines de brevets (même si le facteur $\mathrm{h}$, introduit par Hirsch en 2005 et utilisé trop mécaniquement pour évaluer rapidement les chercheurs n'est pas bien adapté aux indisciplinés) ou simplement du vagabondage scientifique ? Ce n'est pas à l'auteur de juger.

Cette introduction illustre le cheminement de l'auteur, décrit ci-après sur un exemple, sur le comment on passe de formes d'approfondissement disciplinaire à d'autres contextes d'action qui peuvent contribuer à des rapprochements entre le monde de la recherche et la Société. Cette approche «clinique » est une forme de témoignage et n'est sans doute pas, hélas, adaptée à tout... Dans cette publication, petite réflexion sur des éléments créatifs, l'approche initiale a été essentiellement personnelle jusqu'à l'exploitation par d'autres de moyens d'améliorer et d'élargir le procédé initial. Ces autres chercheurs, tout en développant d'autres procédés, n'ont pas remis en cause le principe d'additivité de voxels (volume élémentaire) les uns aux autres pour réaliser un objet conçu par ordinateur. Le succès de cette technologie, aujourd'hui générique avec un marché mondial de l'ordre de 40 milliards $€ /$ an et un taux annuel d'augmentation de l'ordre de $20 \%$ est une chance pour la société, en raison des nombreux avantages que la technologie apporte, mais dans le même temps porte en elle-même ses propres limites, liées en partie à son attractivité. Parmi ces limites, citons une faible reconnaissance scientifique (malgré le caractère un peu magique de la réalisation d'un objet 3D), la faiblesse du potentiel recherche, le besoin de plus en plus impérieux de travailler à plusieurs sur des projets interdisciplinaires, la nécessité de prendre en compte la complexité dans le développement de recherches sur de nouvelles cibles applicatives à fort potentiel économique et social (Impression 4D et Bio-printing). En effet, chaque année, des progrès rendent les technologies $3 \mathrm{D}$ de plus en plus adaptées à une gamme plus large de besoins de production. $\mathrm{A}$ mesure que les vitesses de fabrication d'objets des machines augmentent et que les qualités de surface et les propriétés des matériaux continuent de s'améliorer, des applications commerciales inenvisagées hier s'ouvrent. De plus, le grand changement dans le secteur a eu lieu lorsque les entreprises ont pu/su utiliser la 3D pour produire des pièces finies, réel catalyseur permettant de faire de la fabrication additive ce qu'elle est aujourd'hui. La figure 1 réalisée à partir des données de la Bibliothèque de l'Université de Lorraine illustre le phénomène et met en évidence les origines de certaines inventions en impression 3D.

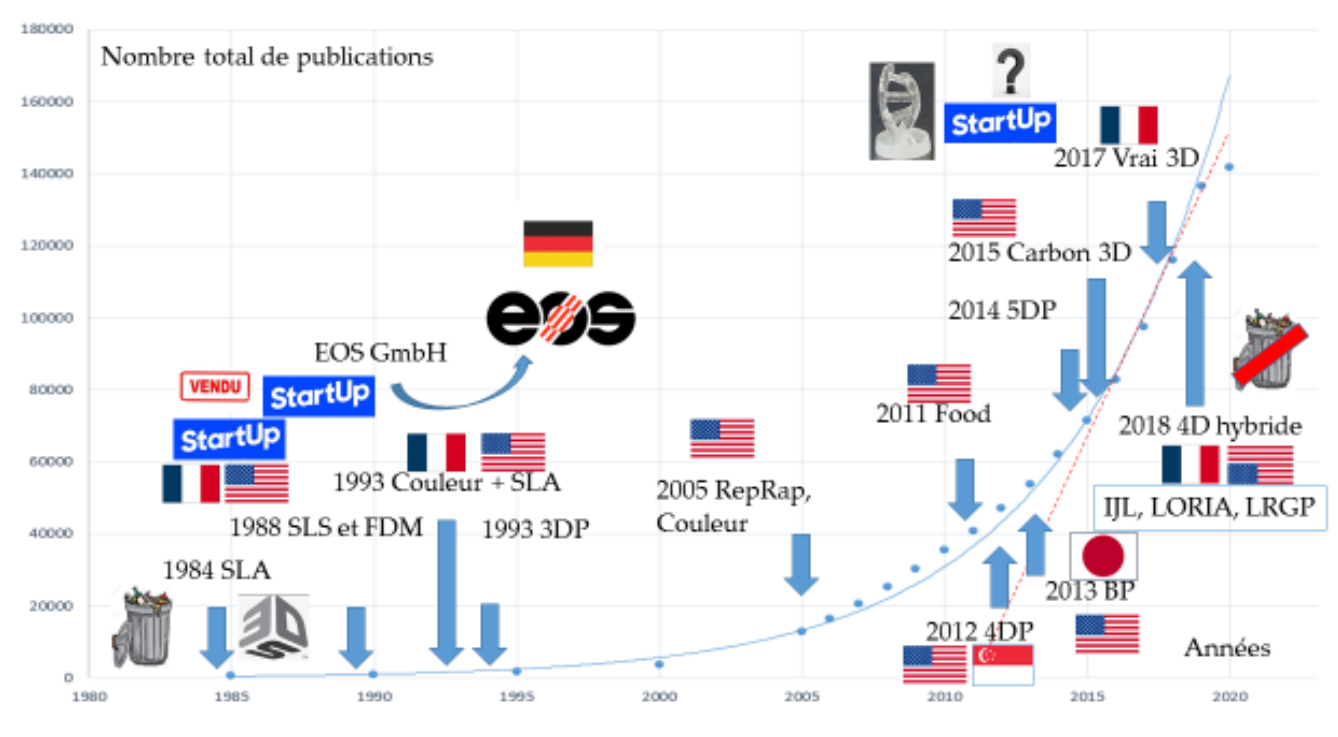

Figure 1. Evolution temporelle du nombre des publications

L'objet de la présente publication est donc multiple. En s'appuyant sur l'histoire de cette technologie à laquelle l'auteur a modestement contribué, ce travail de réflexion se veut d'une part technique, envisageant les avantages singuliers de l'impression 3D en termes divers, les moyens de 
les mettre en situation économique, mais en envisageant les futurs faisant l'objet de travaux scientifiques. D'autre part, il se veut un peu plus épistémologique, sur comment faire science dans des systèmes loin des formations de certitudes initiales des chercheurs, des travaux de recherche inclus dans le court terme, des financements insuffisants, d'équipes à masse sous-critique, etc. Si les potentiels économiques des technologies 3D du futur peuvent atteindre plusieurs milliers de milliards $€ /$ an, si les barrières actuelles sont levées, peut-être faudrait-il aujourd'hui réfléchir au comment faire pour les faire sauter. C'est donc un double chemin qui est offert au lecteur.

Selon ces deux modes de présentation que l'auteur a tenté de découpler, ce qui est proposé, c'est de montrer qu'une solution industrielle est bonne si, et seulement si, son concept, sa démonstration avec des personnes adaptées, une culture d'innovation industrielle, le temps et les financements se rencontrent effectivement. Sans doute qu'en 1984, bien au-dessus de l'auteur, existaient un système d'opinion et de management autocentré, fermé sur lui-même qui n'a même pas pensé à un possible débat sur des ouvertures technologiques futuristes. Cette conformité à une pensée de suivisme industriel et universitaire est de plus en plus considérée comme caduque. Cependant, il existe toujours, en dehors des milieux socio-économiques, chez beaucoup de scientifiques et de gestionnaires une incroyable viscosité : l'attitude la plus commune n'est pas l'ouverture aux autres schèmes explicatifs, mais dans la majorité des cas, l'ignorance et/ou le refus d'accepter leur existence. Il faut faire avec et sans doute développer des ruses qui ne rentraient qu'imparfaitement dans notre éthique et notre culture de chercheurs de l'époque.

\subsection{En préambule : Peut-on aisément sortir du carcan éducatif ?}

En 1966, au cœur des 30 glorieuses, le rationalisme triomphant de l'époque permettait d'envisager que la Société pourrait profiter de tous les progrès de la Science. Ces positions étaient confortées par l'avis des maîtres qui soutenaient l'envie d'un jeune ingénieur de poursuivre ses études par une thèse, petite pierre à un grand ensemble qui, pour l'éternité, resterait toujours en construction. Les choix se sont orientés vers ces maîtres, susceptibles d'associer à cette envie, un financement significatif, c'est à dire déjà un salaire. Ces maîtres disposaient donc d'un statut légitime et détenaient un pouvoir, car ils étaient reconnus et soutenus par la structure dominante. Le laboratoire est un cocon très agréable tant que les règles du jeu ne sont pas toutes connues, axé que l'on est vers la découverte (ou l'impression de la découverte). A l'époque l'argent arrivait, le cadre dans lequel la recherche se développait était très large (en fait, inconnu), limité au peu de temps qu'on pouvait consacrer aux jeunes thésards, réelle chance d'autoformation pour l'auteur. Avec le recul, un poste permanent était octroyé sur dossier sans que la question du retour sur investissement se pose pour la Société. On en est loin aujourd'hui d'entrer aussi facilement au CNRS (André, 2016).

La liberté initiale était grande, en dehors d'un vague rapport annuel à transmettre à un parrain inconnu... La première contrainte réelle est intervenue quand il a fallu participer à la rédaction d'une publication. Il y avait donc des comptes à rendre ! Et à des pairs qui devaient être bien plus savants que le jeune stagiaire de recherche ! Dans cette phase initiatique, on apprend à présenter ses résultats et ses avis de façon à convaincre les rapporteurs de la revue d'accepter votre proposition. Doit-on alors, en paraphrasant Raymond Radiguet, présenter ses résultats pour qu'ils soient compris par son interlocuteur (fût-il masqué) ? L'« Euvre » définitive (!) transmise, on attendait l'avis des experts, sûr de son bon droit, parce qu'ils n'avaient rien compris ou presque, parce qu'ils vous critiquaient. Ce débat difficile, voire tronqué, fonctionnant de façon épistolaire, est toujours difficile, mais finalement cette base permanente de remise en cause est une condition importante du bon accomplissement de la recherche. A l'époque, l'avis de la hiérarchie était sans doute différent du mien, car elle était surtout intéressée par le nombre de publications et par la reconnaissance et la promotion associées.

Dans cet univers douillet, le jeune chercheur disposait du temps, la durée des thèses n'était pas trop strictement limitée, l'argent arrivait (pour lui, ce n'était d'ailleurs pas une question qui se 
posait). Les grandes libertés se sont estompées lentement, avec l'accès à des postes dits de responsabilité, amenant le jeune directeur de recherche au CNRS de l'époque à une situation traditionnelle de compétition scientifique.

Dans ce cadre peu contraint, il a donc été possible de sortir d'un sujet imposé et sans risque (hélas, à faible création d'entropie !), la thèse officielle, pour préparer celle qui n'a jamais été passée, tournée vers d'autres thèmes trouvés plus intéressants parce que choisis. Cette liberté de choix fût plus tard associée à la responsabilité vis-à-vis des thésards. Dans son laboratoire, l'auteur a eu effectivement la chance de disposer d'un réel espace d'initiative, au contraire de ce qui se passe peutêtre dans d'autres contextes où des supérieurs vivent mal l'autonomie de ceux qu'ils encadrent, en particulier lorsque ces derniers possèdent une vision personnelle sur le sens de leur recherche. Pratiquement, à chaque fois, il y eut association entre disciplines et cela s'est passé dans des conditions de confiance et de compréhension souvent peu imaginées au départ (coopérations avec plusieurs équipes du CNRS, de l'INSERM, de l'INRIA de disciplines différentes et avec des partenaires industriels). Ces recherches ont permis d'aller jusqu'à la réalisation de prototypes/procédés industriels sur des aspects capteurs optiques, instruments, logiciels de traitement de l'information, transformations photochimiques et procédés associés, etc.

\subsection{Principe de la fabrication additive avec la stéréo-lithographie laser}

Vint le temps de la stéréo-lithographie laser qui n'est « qu'un » réacteur photochimique très particulier permettant la fabrication d'objets à 3 dimensions par transformation de la matière, résolue dans l'espace. Le développement de cette recherche de fabrication additive a nécessité des connaissances en informatique, en automatique, en optique, en physique et chimie des matériaux, en mécanique, en photochimie, et beaucoup de motivation, de communication et de lobbying pour disposer des financements nécessaires. Quelques partenaires y ont cru, ce qui a permis de passer d'une preuve de concept rudimentaire à des prototypes industriels et à l'attribution d'un prix national de créativité octroyé finalement par toutes les agences de financements (qui avaient oublié préalablement de croire en l'opération). Les brevets initiaux (André, Le Méhauté et de Witte, 1984 ; suivi de plus de 25 autres) ont pour les premiers été mis dans le domaine public par l'industrie associée au CNRS, par méconnaissance du marché potentiel mondial (pour faire simple et un peu langue de bois), même si celui-ci est aujourd'hui de l'ordre de 40 milliards d'euros par an.

C'est la vie, mais quelle vie désordonnée par la pression du temps liée à l'envie de tout explorer : objets en polymères, en métal, en céramiques, procédés divers, utilisation de masques commandés par ordinateur, micro-stéréo-lithographie (quelques micromètres), espace nanométrique (polymérisation par ondes évanescentes, etc.), photographie 3D couleur, fibres et lentilles optiques, etc. Quelle belle expérience, celle dont on parle avec le regret d'être sorti d'une aventure exaltante et riche permise par le foisonnement des idées, un groupe soudé devenu militant, des démonstrations effectives pour explorer d'autres domaines connexes !

Durant ces différentes activités, en particulier en lien avec le monde économique, il n'y a pas possibilité de publier en partie à crédit. L'interaction avec un client, complice parce qu'intéressé, mais ferme sur les engagements, permet une remise en cause quasi-permanente et constitue une obligation de créativité, d'où des thèses, des publications et des brevets. En partant de quelques idées fortes, il a été cependant nécessaire de faire appel à d'autres disciplines que celles maitrisées par l'équipe. Mais, une chance, c'est de connaître les compétences spécialisées de ses collègues. Et de l'exemple présenté ici ressort l'impression que cela a plutôt assez bien marché d'un point de vue recherche!

Avec des amis de la CILAS (Compagnie industrielle des lasers) d'Alcatel, désireux de reprendre à leur compte des travaux issus de Battelle (Adamson, 1968 : Adelman et Lewis, 1971 ; Mc Ginniss et Schwerzel, 1984), une discussion informelle et amicale a conduit à une première réflexion sur la réalisation, par voies photo-physiques et par lasers, d'images 3D par excitation lumineuse (à deux 
photons, issus de deux sources lumineuses), exploitant un processus d'absorption non linéaire. A cette époque, c'était de la belle science, nécessitant des sources lasers impulsionnelles avec d'excellentes propriétés de stigmatisme. En déplaçant les faisceaux laser, par un balayage spatial, une image pouvait être réalisée et, pourquoi pas, l'invention d'une vraie télévision 3D. Après, il a fallu réfléchir un peu. Les relaxations non radiatives se traduisent, peu ou prou, par une élévation de température locale du fluide ou du matériau transparent contenant l'image virtuelle, ce qui fait que les indices de réfraction changent et qu'il est pratiquement impossible d'assurer la rencontre précise des deux faisceaux commandés par des miroirs galvanométriques informatisés (en dehors du premier tir laser). Et en plus, les lasers de l'époque étaient volumineux, coûteux, ce qui ne permettait pas d'envisager une fabrication accessible à l'industrie, et encore moins au grand public.

Dans les brevets de l'Institut Battelle sur l'utilisation de deux faisceaux pour transformer de la matière, conduisant en principe à la réalisation de vrais objets, on pouvait créer des molécules excitées électroniquement par un processus bi-photonique lié à la non-linéarité de l'absorption par utilisation d'impulsions laser intenses. En se décomposant, elles étaient capables d'amorcer des réactions de polymérisation et donc susceptibles de transformer un fluide (le monomère) en un solide (le polymère). Comme dans une construction par éléments, les voxels (briques élémentaires par analogie avec les pixels), on déplaçait (au moins sur le papier) les faisceaux et on construisait de proche en proche l'objet souhaité. Or, l'indice de réfraction des polymères est généralement très différent de celui du monomère qui lui a donné naissance, ce qui rend hasardeuse la réalisation effective d'un vrai objet (de plus de 2 voxels). Même si ceci est possible aujourd'hui, cette aventure paraissait bien compliquée en 1984.

Comment alors réaliser une absorption de lumière résolue dans l'espace si l'on est contraint de respecter la vieille loi de Beer-Lambert d'absorption de la lumière dans un processus monophotonique ? Comment réaliser une économie substantielle en travaillant avec une seule source de photons et contourner les problèmes de changement d'indice par élévation de température, ainsi que l'influence des zones déjà construites par polymérisation ? Les processus à un photon sont bien connus et s'appuient sur un concept simplissime, celui d'épaisseur optique $\mu$ (transmission de $1 / \mathrm{e}$ où e est le nombre d'Euler correspondant à 2,71828 de la lumière incidente à la distance $\mu$ ) qui, en particulier, dépend de la concentration d'un produit absorbant. Pour disposer d'une source peu coûteuse, l'idée de polymérisations radicalaires en chaînes longues était envisageable. En effet, il y a possibilité d'une amplification liée à la réaction, permettant d'utiliser des sources lumineuses de faible puissance. Donc, tout était à notre disposition pour réaliser, couche par couche, un objet réel, à partir de la connaissance des coordonnées de cet objet. Ces coordonnées étaient mémorisées dans un ordinateur qui pilotait des miroirs galvanométriques, pour transformer un liquide en solide par polymérisation d'une couche fluide, voxel après voxel, d'où le concept de fabrication additive. L'ajout d'une deuxième couche, puis d'une troisième, etc. permettait de créer en principe la pièce prototype souhaitée qu'il fallait enfin extraire (et laver) du fluide non photo-transformé. Ces différentes considérations font l'objet de la figure 2 (cf. par exemple Corbel et André, 1994 ; André, 2017).

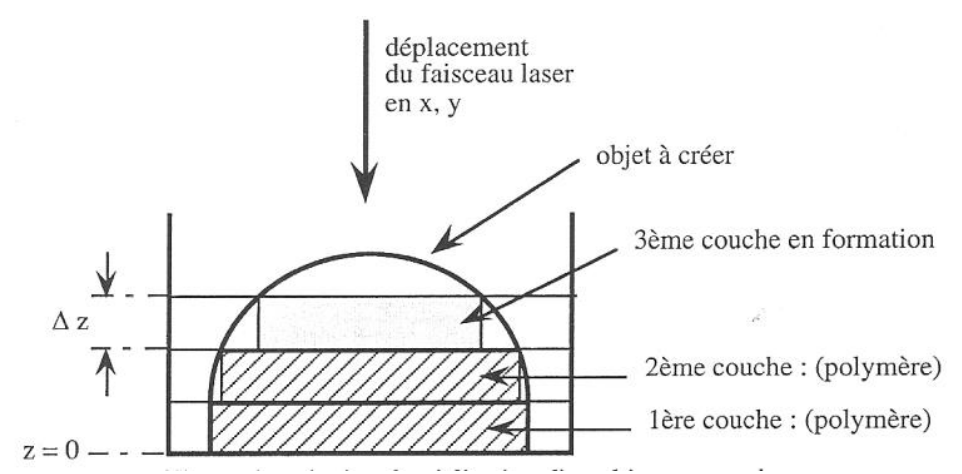

Figure 2. Principe de réalisation d'un objet couche par couche 
D'un point de vue principe technique, la polymérisation se produit sur une faible profondeur là où la lumière atteint la surface, pour autant que l'énergie lumineuse absorbée dépasse un certain seuil. Avec les monomères acryliques, l'oxygène en solution dans ce fluide est un inhibiteur de polymérisation et il convient, pour que la transformation s'opère, de le consommer. La viscosité des fluides utilisés et la densité de flux lumineux sont alors suffisantes pour que la diffusion de l'oxygène issu de zones non éclairées soit sans importance sur la photo-transformation. Ce résultat conduit donc, en principe, à une résolution surfacique meilleure que celle du faisceau laser incident. Pour ce qui concerne la profondeur de pénétration de la lumière, il convient de disposer d'une épaisseur optique $\mu$ légèrement plus grande que celle de la couche placée sur l'objet en construction et d'adapter la puissance du faisceau pour polymériser le fluide en profondeur pour relier entre elles les couches. Un élément non anticipé à l'époque, mais utile pour le procédé, est lié à la présence de l'oxygène de l'air surmontant le liquide photo-réactif. Pour faire simple, il entraine systématiquement l'existence d'une épaisseur non polymérisée de très faible amplitude (de l'ordre du $\mu \mathrm{m}$ ) avec un gradient de polymérisation entre la surface et la couche déjà polymérisée. Quand on superpose une nouvelle couche de fluide, la diffusion de l'oxygène de l'air n'intervient plus entre les deux couches $\mathrm{N}$ et N-1. Quand la photo-polymérisation se développe, à partir de la couche $\mathrm{N}$, il y a accrochage chimique facilité entre les couches, ce qui évite de réaliser des éléments indépendants les uns des autres...

Mais, pour nos tout premiers essais, en l'absence de laser adapté à la réaction photochimique, un samedi qu'il faisait beau à Nancy (si, si...), il a été possible d'utiliser le stigmatisme, certes modeste, de l'astre du jour pour réaliser, avec des masques en papier, de premières expérimentations, tout d'abord avec des monomères acryliques linéaires : le polymère formé étant soluble dans le fluide qui lui donnait naissance, l'échec était cuisant. D'où l'idée d'utiliser des monomères multifonctionnels conduisant effectivement à un matériau strictement insoluble dans son monomère. L'enthousiasme dura tant que furent réalisés par masquage des objets convexes - à base plus large que la partie qui était en construction, comme l'illustre la figure 2. La réalisation, en éclairement solaire modeste, d'un petit pont avec son tablier s'est traduite par deux piles reliées par un volume imprécis : le polymère encore fluide parce qu'en cours de formation était plus dense que le monomère et coulait durant la transformation au fond du récipient. Ainsi, avec ces expériences très préliminaires, deux difficultés étaient cernées, celle du retrait (augmentation de la densité du photo-produit) et la nécessité de placer « au bon endroit » des supports pour que la pièce soit réalisable. (cf. figure 3).

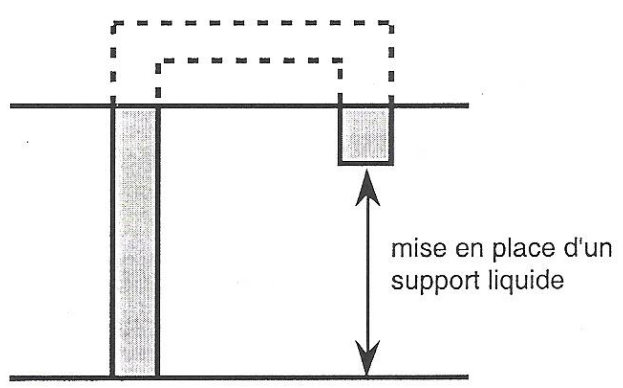

Figure 3. Illustration de la difficulté de construire un objet sans support

Il y avait donc nécessité de jouer avec les monomères et/ou les oligomères, les amorceurs photochimiques et leur viscosité (pour la réalisation correcte des couches successives), en même temps qu'il fallait réaliser des supports faciles à retirer lors de la création de la pièce. Le problème du retrait volumique lié à la polymérisation peut en partie être traité par un choix adapté de monomères, mais plutôt grâce à un procédé qui permet une relaxation volumique de voxels indépendants, qu'il est possible, dans un deuxième passage du faisceau laser, de relier entre eux. De plus, il n'est pas nécessaire de polymériser tout le volume de la pièce à construire, la surface et des renforts peuvent suffire pour lui donner la forme souhaitée, avec la difficulté de définir un procédé 
automatisé quelle que soit la forme de la pièce à construire. La transformation globale de l'objet peut, dans une dernière phase être réalisée dans un système d'irradiation classique qui transforme tout le fluide résiduel en solide. Ainsi fut fait avec des lasers UV ou IR et des pièces commencèrent à sortir pour notre grande satisfaction. Au début, elles disposaient d'une espérance de vie modeste à cause de tensions internes évoluant dans le temps dans le matériau (non optimisé) qui conduisaient à des ruptures. Plus de 25 brevets furent pris (dont le premier, le plus important, 15 jours avant nos concurrents américains). Jouer sur le procédé, le choix des sources lumineuses, les matériaux et leurs formulations, le choix des amorceurs photochimiques, la commande informatisée de miroirs galvanométriques et piloter la réalisation d'une pièce relevait d'un exercice d'acrobatie intellectuelle qui dépassait un peu l'interdisciplinarité classique parce que les soutiens (humains et financiers) étaient plutôt dispensés avec modération, mais pas uniquement. Cela ressemblait à du bricolage et, au moins au début, assez artisanal, mais il s'est agi d'une recherche apprenante.

D'ailleurs, la première publication a été refusée parce que le concept n'existait pas et que l'approfondissement disciplinaire était effectivement modeste. Les soutiens financiers de l'Etat ne sont venus qu'avec parcimonie parce personne n'a cru à cette technologie émergente ; seule la Région de Lorraine est restée en support de l'avancement du projet avec, en association, des reconnaissances sous formes de contrats industriels d'horizons divers. C'est au fond parce que ce système qui est une photocopieuse 3D était attractif pour les milieux socio-économiques que l'équipe était courtisée, parce que cette méthode devait faciliter la réalisation de pièces prototypes coûteuses impossibles à réaliser ou nécessitant autrement des semaines de travail (cf. figure 4). Il n'était donc pas nécessaire de disposer d'un business plan pour convaincre. Mais, dans le même temps, si le groupe de recherche disposait de la propriété intellectuelle des systèmes qu'elle développait, il devenait de plus en plus difficile de mettre en place une structure industrielle crédible et robuste, malgré plusieurs startups qui finalement ont été rachetées par l'entreprise américaine arrivée seconde dans la compétition initiale pour le dépôt du premier brevet, les propriétaires des brevets étant de plus en plus nombreux...

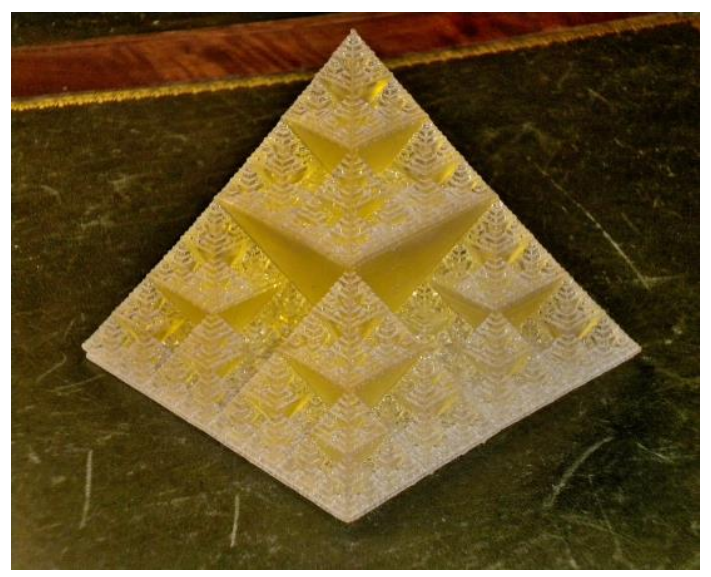

Figure 4. Exemple d'objet non réalisable par des voies classiques : une pyramide fractale

Le début du XXIe siècle est marqué par la présence hégémonique de la transition numérique avec, en complément technologique et pratique, les procédés de fabrication additive, susceptibles d'affecter en profondeur et rapidement la société occidentale (André, 2019). «Face à des marchés d'innovation radicale, où les premiers arrivés peuvent acquérir des positions dominantes décisives, et faire disparaître au passage d'autres marchés et les acteurs économiques installés, rester en retrait et voir venir peut entraîner des coûts économiques et sociaux considérables » (France-Stratégie, 2017). 


\section{2- La stéréo-lithographie}

Dans le paragraphe 1.2 précédent, le passage de l'idée à une preuve de concept a été réalisé rapidement avec des verrous scientifiques et technologiques qui constituent encore l'ossature des recherches actuelles dans le domaine. Le présent paragraphe tente de présenter la technologie avec quelques éléments spécifiques, cependant synthétisés pour cet article. Le lecteur intéressé par cette rubrique pourra consulter en particulier l'ouvrage publié en 2017 par l'auteur.

Première technologie d'une série, la stéréo-lithographie est une des technologies de la fabrication additive. Le terme «impression 3D » (cf. le site http://play.tojsiab.com/bUV0ckUtdFBBRWsz) recouvre en réalité toute une série de procédés qui ont en commun de fabriquer des objets par dépôt de couches successives extrêmement fines de matière [ou plus simplement d'ajouts comme des fils fondus], lesquelles sont solidifiées au fur et à mesure par une source d'énergie (laser, par exemple) [...]. Elle est définie par l'AFNOR (2011) comme «l'ensemble des procédés permettant de fabriquer, couche par couche, par ajout de matière, un objet physique à partir d'un objet numérique ». Elle permet d'explorer plusieurs axes spécifiques tels que ceux présentés sur la figure 5 issue de Conner et al (2014).

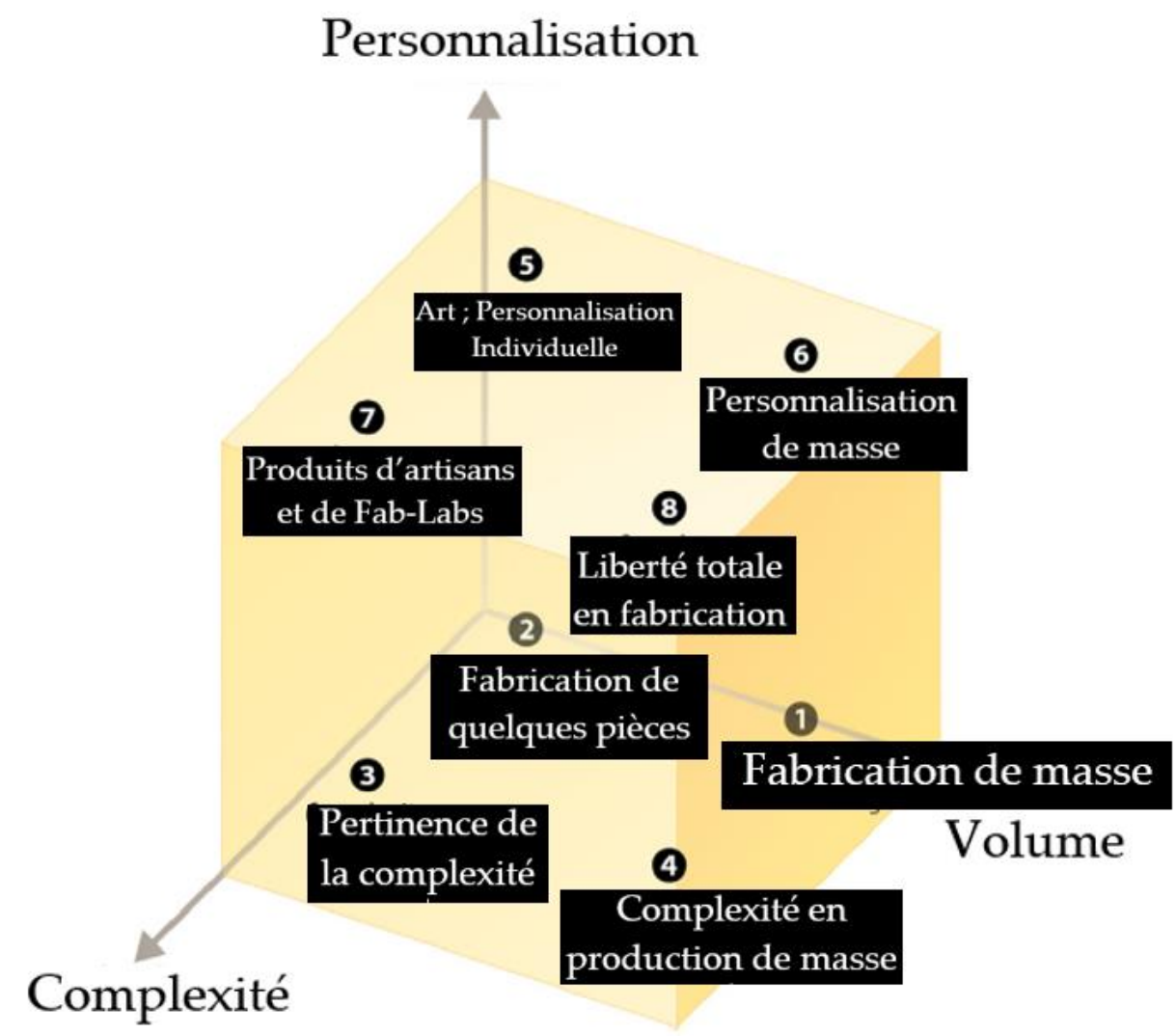

Figure 5. Axes d'intervention de la fabrication additive

«L'impression 3D est ainsi considérée - avec l'Internet mobile, l'Internet des objets, le cloud computing, le Big Data, l'automatisation des métiers du savoir, la robotique de pointe ou encore les matériaux avancés - comme l'une des technologies liées au numérique susceptible de transformer profondément [...] les modes de production et, par conséquent, les modèles économiques actuels » (Ingelaere, 2015). Il s'agit, au sens de Star et Griesemer (2008) d'un nouvel objet-frontière, que l'on parle d'impression 3D, de photocopie 3D, de 3D printing ou de fabrication additive... ( «Suffisamment plastique pour s'adapter aux besoins locaux et aux contraintes des divers groupes qui l'utilisent, tout en étant suffisamment robuste pour maintenir une identité commune d'un site à l'autre » (Ingelaere, 2015)). 
La figure 6 extraite de Bouffaron (2014) rappelle les différentes étapes impliquées dans le procédé de fabrication additive. C'est sur la connaissance du trajet du faisceau lumineux pour une cote donnée que l'objet se construit à partir du déplacement informatisé du faisceau laser. De proche en proche, couche après couche, l'objet se construit.

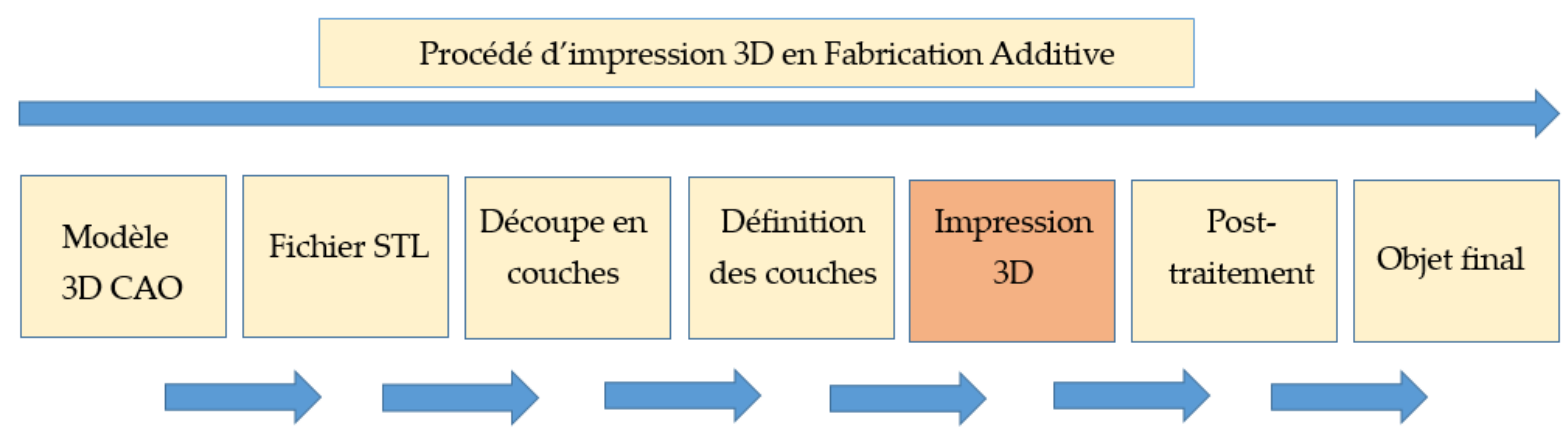

Figure 6. Différentes étapes de la fabrication additive

\subsection{Interaction lumière-matière et polymérisation résolue dans l'espace}

L'absorption mono-photonique suit, dans des milieux transparents, la loi de Beer-Lambert. Localement, pour une concentration c donnée de la substance absorbante, l'intensité lumineuse absorbée est proportionnelle au produit c. $\exp (-\varepsilon . c . x)$ où $\mathrm{x}$ est la distance dans la cuve de la fenêtre d'entrée de la lumière et $\varepsilon$ un coefficient appelé cœfficient d'extinction moléculaire. Elle s'exprime par (André, 2017) :

$$
\mathrm{Ia}(\mathrm{x})=\varepsilon \cdot c \cdot \mathrm{F}(0) \cdot \exp (-\varepsilon \cdot c \cdot \mathrm{x})
$$

Où $\mathrm{F}(0)$ est la densité de flux de photons à la longueur d'onde considérée. On désigne également par $\mu$ l'épaisseur optique définie par :

$$
\mu=1 / \varepsilon . \mathrm{c}
$$

Déjà, à partir de cette base, on imagine que si e est l'épaisseur d'une couche à polymériser, il faudra choisir $\mu$ comme proche de e. $\varepsilon$ est une donnée qui dépend de la substance absorbante, allant de quelques dizaines d'unités à $10^{5}$ dans les unités classiques des physico-chimistes et des spectroscopistes $\left(\mathrm{M}^{-1} .1 . \mathrm{cm}^{-1}\right.$ où $\mathrm{M}$ désigne des moles, 1 des litres). Pour des épaisseurs typiques de l'ordre de $0.1 \mathrm{~mm}$, les concentrations d'amorceur seront en général supérieures à $10^{-3} \mathrm{M} \cdot \mathrm{l}^{-1}$. Dans ces conditions, l'essentiel du mélange réactif sera constitué d'un monomère pur ou d'un mélange de monomères engagé dans la transformation souhaitée liquide-solide.

Remarque : Milieu chargé

De nombreuses applications impliquent des milieux chargés (réalisation de céramiques par exemple); dans ces conditions, la lumière est l'objet de diffusion ce qui entraine des modifications de l'épaisseur optique (même si en première approximation, la transmission de l'énergie lumineuse suit une loi proche de celle de Beer-Lambert (Balland, Guillard et André, 1984 ; Braun et André, 1985). Cette épaisseur de pénétration Ep est définie, en l'absence d'absorption par la résine, de manière approximative par :

$$
\mathrm{Ep}=2 . \mathrm{D}_{50} /(3 . \mathrm{Q} . \mathrm{C})
$$

où les symboles suivants représentent :

- $\mathrm{D}_{50}$ : le diamètre moyen des particules (supposées sphériques);

- $\mathrm{Q}$ : paramètre de diffusion de la lumière défini par $h . \Delta \mathrm{n}^{2} / \lambda$ avec : 
- $\mathrm{h}$ : distance inter-particulaire ;

- $\lambda$ : longueur d'onde d'irradiation :

- $\Delta \mathrm{n}:$ l'écart des indices de réfraction entre la résine et la charge ;

- $\mathrm{C}$ : la densité de charge.

Avec l'amorceur, si $\mu$ est l'épaisseur optique en l'absence de charge, l'épaisseur $\mu_{\mathrm{c}}$ en présence d'additifs solides s'exprime par la relation approchée suivante :

$$
1 / \mu_{\mathrm{c}}=1 / \mu+1 / \mathrm{Ep}
$$

Deuxième aspect, les résines contiennent des groupements absorbant dans l'ultra-violet lointain, ce qui impose des choix de molécules qui vont amorcer la réaction de polymérisation dans une fenêtre spectrale libre. On utilise généralement pour des réactions en chaînes des cétones qu'on excite dans l'UV moyen (autour de $350 \mathrm{~nm}$ ), plutôt que des composés plus complexes absorbant dans le visible, à cause de de risques de polymérisations parasites induites par la lumière du jour (même si des objets ont pu être réalisés il y a plus de vingt ans, dans le visible par Bertsch, 1996).

Ces composés excités électroniquement peuvent se fragmenter en radicaux libres R. qui vont être susceptibles d'amorcer une polymérisation radicalaire. La figure 7 suivante (Susperregui, 2010 ; Decker, 2002; André et Corbel, 1994) représente schématiquement une réaction, avec son mécanisme de polymérisation radicalaire en chaînes qui permet à partir d'un radical créé par un processus photonique de transformer un grand nombre de molécules.

\begin{tabular}{|cc|}
\hline Etape d'amorçage (photochimique) & $\mathrm{A} \rightarrow 2 \mathrm{R}$. \\
Propagation (M pour monomère) & $\mathrm{R} .+\mathrm{M} \rightarrow \mathrm{R} .\left(\mathrm{k}_{1}\right)$ \\
Terminaison (dimère) & $\mathrm{R} .+\mathrm{R} . \rightarrow \mathrm{R}_{2}$ \\
Terminaison (piègeage) & $\mathrm{R} . \rightarrow(\mathrm{R}).\left(\mathrm{k}_{2}\right)$ \\
\hline
\end{tabular}

Figure 7. Mécanisme radicalaire de polymérisation en chaînes

Compte tenu de la concentration des monomères (milieu quasi-pur en monomère au moment de l'amorçage), la polymérisation a lieu de proche en proche, à l'échelle moléculaire comme l'indique la figure 8. Si l'on dispose de surcroît de monomères multifonctionnels, le polymère qui se construit est normalement insoluble dans le monomère qui lui a donné naissance (cf. André et Corbel, 1994).

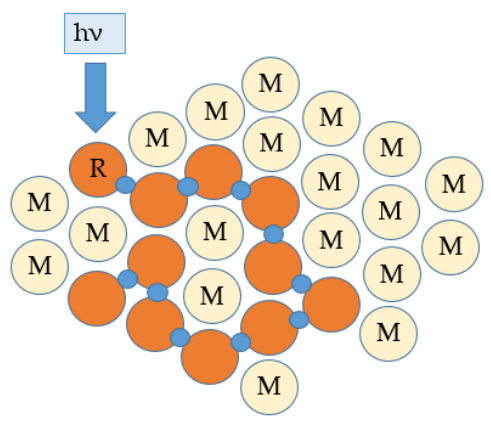

Figure 8. Polymérisation par proximité spatiale (hv représente l'énergie du photon où v est la fréquence de la radiation et $h$ la constante de Planck)

D'un point de vue cinétique, la transformation suit une loi d'allure sigmoïde telle que présentée sur la figure 9, avec une cinétique initiale lente dans la zone I liée à la présence d'inhibiteurs de réaction (hydroquinone par exemple pour autoriser la conservation des monomères, oxygène en 
solution, etc.) qui sont lentement consommés (Andrzejewska, 2001). La zone II représente une réaction dans un espace libre de polymérisation, alors que dans la zone III, celle-ci est de plus en plus gênée par le polymère tridimensionnel qui se forme et qui limite l'espace accessible à la réaction de macro-radicaux avec des monomères restants, et donc la cinétique réactionnelle (Irmouli, George et Merlin, 2009 ; Andrzejewska, 2001). Expérimentalement, il est possible de montrer que dans cette zone des radicaux libres sont présents, mais sont emprisonnés dans la matrice polymérique. Si l'amorceur participe à la réaction de consommation des inhibiteurs, il convient de disposer d'une concentration de cette substance qui doit être supérieure à celle des inhibiteurs (sinon, il y aurait risque d'une non polymérisation ou d'une profondeur de polymérisation différente de celle de la consigne). L'oxygène, dans les réactions radicalaires de polymérisation est souvent l'inhibiteur principal ; sa concentration dans les fluides organiques est de l'ordre de $10^{-2} \mathrm{M}^{-1}{ }^{-1}$. Dans ces conditions, si l'on souhaite disposer par exemple d'une valeur de $\mu$ de $0.1 \mathrm{~mm}$, avec c $>0.01$ M.1 ${ }^{-1}$, la valeur de $\varepsilon$ doit être inférieure à $10^{4} \mathrm{M}^{-1} \cdot 1 . \mathrm{cm}^{-1}$, ce qui permet de calibrer le choix d'un amorceur photochimique acceptable relativement à la fabrication souhaitée (cf. Mokbel, 2015 pour des sélections d'amorceurs ; Stampfl et al, 2008 ; Delaire et al, 2016).

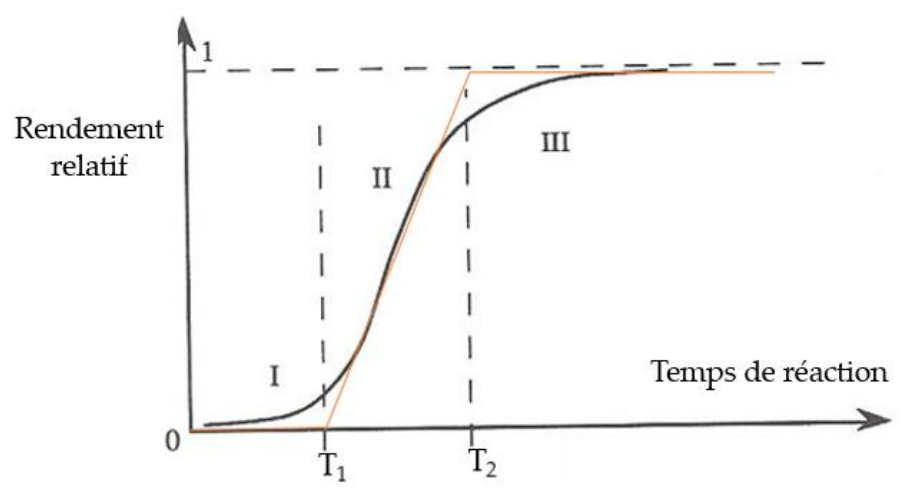

Figure 9. Courbe cinétique de polymérisation type

En choisissant la longueur d'onde, l'amorceur et la résine, il est possible de disposer d'une panoplie considérable de choix, avec aujourd'hui par les résines commerciales qui ont fait leurs preuves.

\subsubsection{Mise en place des couches}

Un objet en cours de réalisation est descendu dans la cuve contenant la résine pour pouvoir être surmonté par du fluide réactif et remonté de manière à ce que le fluide dépasse de $\Delta \mathrm{z}$ l'objet en construction de la manière la plus horizontale possible. Si le fluide réactif est faiblement visqueux, la mise en équilibre pour une remontée rapide de l'objet en construction va être également rapide ; en revanche, pour un oligomère plus visqueux, comme une résine pré-polymérique, ce temps de mise en équilibre peut être beaucoup plus long, parfois rhédibitoire. Dans des conditions d'écoulement laminaire, sans artifice extérieur, cette hauteur décroit sensiblement de manière exponentielle avec des temps de relaxation qui peuvent dépasser la minute. Le problème lié à la mise en place des couches est donc un facteur important à prendre en considération dans ce procédé de stéréo-lithographie. En présence de mouillage total, il peut être utile de garder en mémoire la loi de Tanner qui exprime, dans un système à relaxation libre, une relaxation qui est proportionnelle au temps à la puissance -3/10 (De Gennes, Brochart-Wyart et Quéré, 2005). Avec des milieux très visqueux, ce temps de relaxation peut être inacceptable dans un procédé industriel où l'investissement financier, appliqué au temps de fabrication d'un objet, peut devenir un critère de coût de réalisation d'un objet, fut-il compliqué. Depuis peu, Carbon 3D (2019) a trouvé une astuce pour pallier en grande partie ce problème. 
Dans Corbel et André(1994), une première voie avait été trouvée pour accélérer cette relaxation temporelle en faisant remonter l'objet au-delà de la cote finale. D'autres techniques utilisant des râcleurs mécaniques sont aujourd'hui utilisées avec succès, mais pour autant, pour des couches d'épaisseurs modestes, de l'ordre de quelques dizaines de $\mu \mathrm{m}$, il convient de ne pas détruire avec le râcleur, l'objet en cours de construction (évitement de cisaillements critiques). Ainsi, tout procédé réduisant ou éliminant la mise en place de couches est le bienvenu car permettant de travailler plus sereinement avec des oligomères, éventuellement chargés et fortement visqueux.

\subsubsection{Intervention de supports durant la construction de l'objet}

Supposons une pièce à réaliser de forme complexe du type de l'objet présenté sur la figure 10 , si la pièce en construction n'est pas supportée à la hauteur $\mathrm{z}_{0}$, l'objet final ne sera jamais conforme à la prévision numérique (cf. par exemple, Mirzendehdel et Sureh, 2016). Il faut donc envisager de la tenir par l'introduction de supports qui seront à éliminer après fabrication.

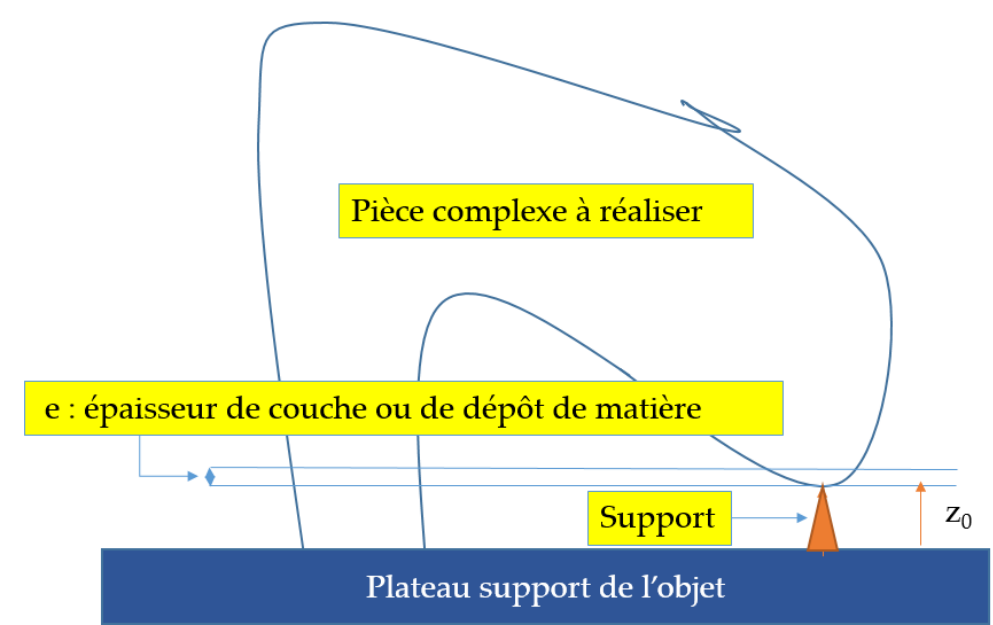

Figure 10. Nécessité de mise en place de supports pour des pièces complexes (dépendant du procédé utilisé)

Dans ces conditions particulières, il est nécessaire d'adjoindre à la numérisation de l'objet des additifs complémentaires qui en permettent sa faisabilité. En fin de fabrication, comme pour la base, il y aura nécessité d'éliminer les éléments d'échafaudage par des voies physiques ou chimiques (cf. supra). Ce principe étant défini, si la pièce est creuse, il sera probablement délicat d'espérer éliminer des supports internes par des méthodes mécaniques, ce qui conduit à travailler avec des machines spéciales adaptées à des multi-matériaux ou ne nécessitant pas de supports (Shusteff et al, 2017 ; Loterie, Delrot et Moser, 2019 ; Kelly, 2019 ; André, Gallais et Amra, 2016).

\subsubsection{Interventions manuelles}

De manière générale, la réalisation d'un objet imprimé en 3D nécessite un modèle numérique tridimensionnel réalisé à l'aide de logiciels de CAO (Conception Assistée par Ordinateur). Une fois ouverts dans un logiciel préparant l'impression, ils sont analysés et transformés en instructions envoyées à l'imprimante (étape, dite de slicing) spécifique dans le processus de fabrication additive. L'addition localisée et progressive de matière/d'énergie par couches successives construit graduellement l'objet physique. Ce processus réalisé, des opérations de post-production peuvent être nécessaires en fonction des matériaux utilisés et de la qualité finale désirée (Corbel et André, 1994 ; Cotteleer 2014 ; Cotteleer, Holdowsky et Mahto, 2016). Ces différentes étapes sont représentées sur la figure 11 (elles sont applicables à la plupart des procédés). 


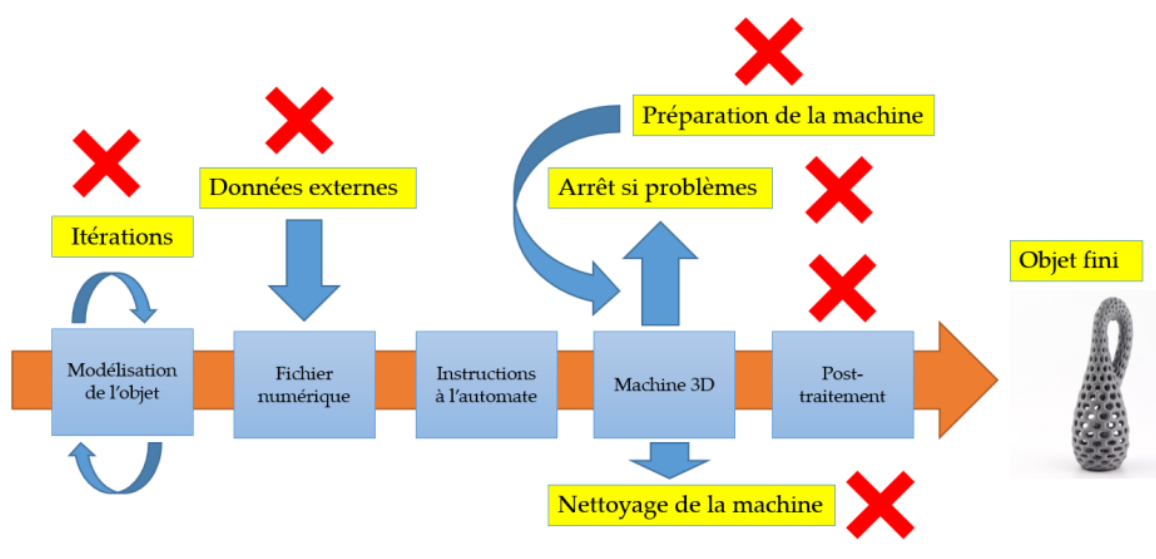

Figure 11. Etapes de fabrication d'un objet par fabrication additive (croix rouge : intervention humaine dans le processu)

Comme on peut le constater à l'examen de cette figure, on est loin de ce que fait un utilisateur moyen avec une imprimante classique, à cause d'un nombre élevé d'interventions humaines...

\subsection{Les autres grandes technologies de fabrication additives}

Les technologies de la fabrication additive se développent avec cinq préoccupations principales présentes et engagées vers des aspects plus prospectifs :

- Montrer que la fabrication additive s'inscrit dans un processus industriel permettant de réduire le « time-to-market »;

- Trouver de nouveaux débouchés à la technologie: du jeu pour enfants (Mattel®) à l'impression d'organes ;

- Innover pour maintenir la dynamique et durer : approche incrémentale sur des procédés nouveaux et surtout des matériaux et multi-matériaux ; approche ruptionnelle en reprenant le concept (exemple du 4D printing);

- Réaliser des preuves de concept et communiquer ; les démonstrateurs initiaux se développent dans des structures lègères avec un investissement modeste mais avec une forte créativité ;

- Fabriquer des produits finis en un nombre d'étapes le plus limité possible (André, 2017).

Compte tenu de cette feuille de route, le champ couvert est très large, et clairement une seule technologie ne peut être à même de le couvrir. Ainsi, si, au départ, la stéréo-lithographie, a servi de démonstrateur pour illustrer l'intérêt du concept, d'autres technologies ont émergé, disposant chacune d'avantages et d'inconvénients, non seulement entre-elles, mais également vis-à-vis d'autres technologies complémentaires ou concurrentes (fabrication soustractives par exemple). Aujourd'hui, la palette technologique est plus métissée que jamais avec des services et des applications qui demandent à être agencés de façon spécifique suivant la capacité créative de chacun ou du besoin industriel.

Selon Vaezi, Seitz et Yang (2013), les technologies 3D (par addition de couches, ou plus généralement de matière) sont importantes en nombre et peuvent être représentées par les différents thèmes rassemblés figure 12 (cf. également MADEELI, 2017 ; Murr et Johnson, 2017 ; Goh et al, 2017 ; Bhushan et Caspers, 2017) et sur le tableau 1 interprété de Hague, Reeves et Jones (2016), Bouffaron (2014) et de Brethome et al (2016). Les principes sont sensiblement identiques et les procédés différent essentiellement par les modes d'adhésion de la matière à l'objet en construction (cf. par exemple, Mizeret, 2015 ; Robinson, 2015 ; Verhulst, 2015). Néanmoins, si la règle du jeu est bien de disposer, soit d'une énergie localisée, soit d'un positionnement du matériau localisé, soit des deux, il paraît nécessaire d'engager une analyse un peu plus fine sur ces différents procédés pour examiner ensuite leurs spécificités applicatives. Le lecteur intéressé par ces différentes technologies pourra trouver des informations dans André (2017). 


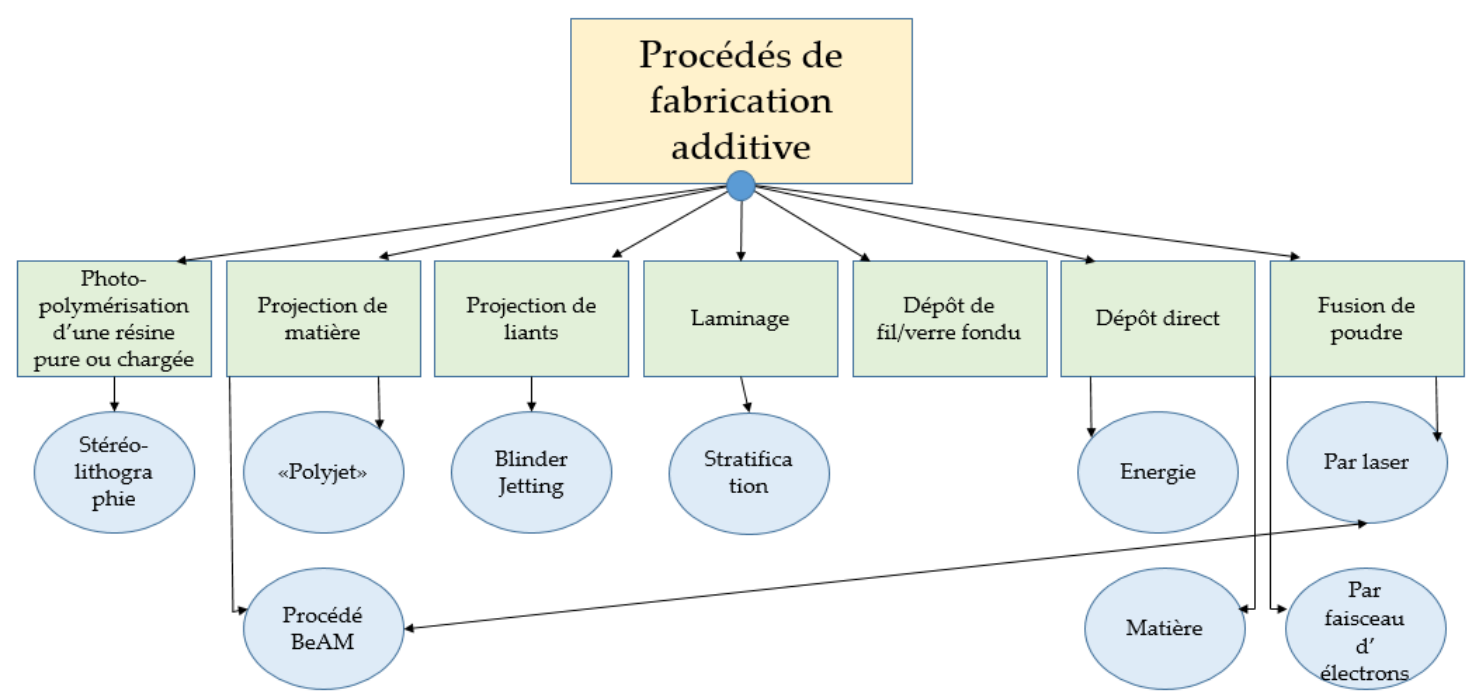

Figure 12. Les différents procédés de fabrication additive

\begin{tabular}{|c|c|c|c|c|c|c|}
\hline Procédé & Energie & Matière & Principe & $\begin{array}{l}\text { Nature des } \\
\text { liaisons }\end{array}$ & $\begin{array}{c}\text { Post- } \\
\text { traitement }\end{array}$ & Spécificités \\
\hline $\begin{array}{l}\text { Stéréo- } \\
\text { lithographie }\end{array}$ & Lumière & Monomère & $\begin{array}{l}\text { Réaction } \\
\text { chimique }\end{array}$ & $\begin{array}{l}\text { Photo- } \\
\text { polymérisation } \\
\text { résolue dans } \\
\text { l'espace }\end{array}$ & $\begin{array}{l}\text { Post- } \\
\text { polymérisation } \\
\text { Nettoyage } \\
\text { Elimination des } \\
\text { supports }\end{array}$ & $\begin{array}{l}\text { Absorption } \\
\text { mono et bi- } \\
\text { photonique } \\
\text { Supports } \\
\text { Du } \mu \text { m au m } \\
\text { Problèmes de } \\
\text { vieillissement } \\
\text { et de retrait } \\
\text { Possibilité de } \\
\text { travailler avec } \\
\text { des matériaux } \\
\text { chargés }\end{array}$ \\
\hline $\begin{array}{l}\text { Projection } \\
\text { de matière } \\
\text { (1) }\end{array}$ & Lumière & $\begin{array}{l}\text { Goutte de } \\
\text { monomère } \\
\text { liquide; de } \\
\text { poudre } \\
\text { fusible }\end{array}$ & $\begin{array}{l}\text { Réaction } \\
\text { chimique }\end{array}$ & $\begin{array}{l}\text { Photo- } \\
\text { polymérisation } \\
\text { Fusion de } \\
\text { poudre }\end{array}$ & $\begin{array}{l}\text { Ebarbage } \\
\text { Post- } \\
\text { polymérisation }\end{array}$ & $\begin{array}{l}\text { Grand choix de } \\
\text { matières }\end{array}$ \\
\hline $\begin{array}{l}\text { Projection } \\
\text { de matière } \\
\text { (2) }\end{array}$ & Sans & $\begin{array}{l}\text { Goutte d'un } \\
\text { matériau } \\
\text { fondu }\end{array}$ & Solidification & $\begin{array}{l}\text { Collage résolu } \\
\text { en surface }\end{array}$ & Cf. supra & Cf. supra \\
\hline $\begin{array}{l}\text { Projection } \\
\text { de liants }\end{array}$ & $\begin{array}{ll}\text { Agent } & \text { de } \\
\text { liaison } & \end{array}$ & $\begin{array}{l}\text { Matériau } \\
\text { pulvérulent }\end{array}$ & $\begin{array}{l}\text { Réaction de } \\
\text { collage }\end{array}$ & $\begin{array}{l}\text { Collage résolu } \\
\text { en surface }\end{array}$ & $\begin{array}{ll}\text { Elimination } & \text { de } \\
\text { la poudre en } & \text { excès }\end{array}$ & $\begin{array}{l}\text { Pas de supports } \\
\text { Couleurs } \\
\text { multiples } \\
\text { Fragilité des } \\
\text { objets }\end{array}$ \\
\hline Laminage & $\begin{array}{l}\text { Collage } \\
\text { soudure }\end{array}$ & Feuille & $\begin{array}{l}\text { Découpe } \\
\text { résolue en } \\
\text { surface }\end{array}$ & Collage & $\begin{array}{l}\text { Séparation des } \\
\text { feuilles }\end{array}$ & $\begin{array}{l}\text { Objet } \\
\text { anisotrope } \\
\text { Peu coûteux }\end{array}$ \\
\hline $\begin{array}{ll}\text { Dépôt } & \text { de } \\
\text { verre/fil } & \\
\text { fondu } & \end{array}$ & Sans & $\begin{array}{l}\text { Matière } \\
\text { fondue }\end{array}$ & Solidification & $\begin{array}{l}\text { Collage résolu } \\
\text { en surface }\end{array}$ & $\begin{array}{l}\text { Elimination des } \\
\text { supports }\end{array}$ & $\begin{array}{l}\text { Précision } \\
\text { Qualité } \\
\text { surface } \\
\text { Peu coûteux }\end{array}$ \\
\hline $\begin{array}{l}\text { Dépôt direct } \\
\text { Fusion de }\end{array}$ & Laser & $\begin{array}{l}\text { Matériau } \\
\text { pulvérulent }\end{array}$ & Solidification & $\begin{array}{l}\text { Collage résolu } \\
\text { en surface }\end{array}$ & $\begin{array}{l}\text { Elimination de } \\
\text { la poudre en }\end{array}$ & $\begin{array}{l}\text { Etat de surface } \\
\text { améliorable }\end{array}$ \\
\hline
\end{tabular}




\begin{tabular}{|l|l|l|l|l|l|l|}
\hline poudre (1) & & & & excès & $\begin{array}{l}\text { Grand choix de } \\
\text { matériaux }\end{array}$ \\
\hline $\begin{array}{l}\text { Dépôt direct } \\
\text { Fusion de } \\
\text { poudre (2) }\end{array}$ & $\begin{array}{l}\text { Source } \\
\text { électronique }\end{array}$ & $\begin{array}{l}\text { Matériau } \\
\text { pulvérulent }\end{array}$ & Solidification & $\begin{array}{l}\text { Collage résolu } \\
\text { en surface }\end{array}$ & $\begin{array}{l}\text { Elimination de } \\
\text { la poudre en } \\
\text { excès }\end{array}$ & Cf. supra \\
\hline $\begin{array}{l}\text { Dépôt direct } \\
\text { Fusion de } \\
\text { poudre (3) }\end{array}$ & IR + Masque & $\begin{array}{l}\text { Matériau } \\
\text { pulvérulent }\end{array}$ & Solidification & $\begin{array}{l}\text { Collage résolu } \\
\text { en surface }\end{array}$ & $\begin{array}{l}\text { Elimination de } \\
\text { la poudre en } \\
\text { excès }\end{array}$ & Cf. supra \\
\hline
\end{tabular}

Tableau 1. Les différentes méthodes de fabrication additive : quelques éléments de différenciation

Ainsi, aujourd'hui il est possible de réaliser des objets en polymères, en céramiques, en métal, en ciment avec des tailles qui vont de quelques dizaines de nanomètres jusqu'à plusieurs dizaines de mètres.

\section{Les attractivités de la fabrication additive}

Après la machine à vapeur, l'électricité et l'automatisation, la « robolution » est la quatrième révolution industrielle incluant la fabrication additive (cf. Moussion, 2015). Le concept d'Industrie 4.0 correspond à une nouvelle façon d'organiser les moyens de production : l'objectif est la mise en place d'usines dites intelligentes capables d'une plus grande adaptabilité dans la production et d'une allocation plus efficace des ressources, ouvrant ainsi la voie à une nouvelle révolution industrielle (Valero, 2016). Après l'automatisation, cette nouvelle révolution serait fondée sur l'usine intelligente, caractérisée par une interconnexion des machines et des systèmes au sein des sites de production mais aussi entre eux et l'extérieur (clients, partenaires, autres sites de production). L'industrie 4.0 correspond, en quelque sorte, à la numérisation de l'usine. Elle a recours à l'Internet des objets, aux systèmes cyber-physiques et à la fabrication additive, c'est-à-dire aux réseaux virtuels servant à contrôler des objets physiques. Alors, l'usine intelligente se caractérise par une communication continue et instantanée entre les différents outils et postes de travail intégrés dans les chaînes de production et d'approvisionnement. La figure 15 issue d'OECD (2017) place la fabrication additive à l'intérieur du concept d'Industrie 4.0 (cf. par exemple, André, 2019).

Mais la fabrication additive ne se limite pas à l'entreprise, elle dispose de champs d'usage, d'interactions et d'utilisateurs beaucoup plus vastes (cf. DGE, 2016). Le tableau 4 (Rayna et Striukova, 2016) indique les étapes, les transitions entre les différents développements de la technologie depuis la création du concept jusqu'à des applications industrielles et domestiques. Il met en évidence des temporalités assez longues de l'ordre de la génération humaine (ce qui n'a pas été le cas du transistor, mais qui rentre assez bien dans les dynamiques classiques de l'innovation).

\begin{tabular}{|c|c|c|c|c|c|}
\hline Adoption & Début & Design & Outillage & Fabrication & Distribution \\
\hline $\begin{array}{c}\text { Prototypage } \\
\text { rapide }\end{array}$ & Début 1990 & & & & \\
\hline $\begin{array}{c}\text { Outillage } \\
\text { rapide }\end{array}$ & Fin 1990 & & & & \\
\hline $\begin{array}{c}\text { Fabrication } \\
\text { directe }\end{array}$ & Fin 2000 & & & & \\
\hline $\begin{array}{c}\text { Fabrication } \\
\text { domestique }\end{array}$ & Début 2010 & & & & \\
\hline
\end{tabular}

Tableau 2. Etapes d'adoption des technologies d'impression 3D

L'impression 3D peut remettre en cause le concept de chaîne logistique et ses conséquences pourraient être nombreuses : production en flux tendus, réduction du stockage, du transport, des 
énergies et des coûts associés (pour autant qu'on puisse réaliser des objets en bonne matière). Selon Branche (2015), « la totalité de cette chaîne devient même obsolète. Les possesseurs d'imprimantes $3 \mathrm{D}$ n'ont qu'à modéliser leur pièce ou récupérer un fichier $3 \mathrm{D}$ pour fabriquer leur propre objet, court-circuitant ainsi tous les intermédiaires habituels. C'est ainsi que la philosophie basée sur le « Do it yourself », l'open source, c'est-à-dire le partage de l'information, alliée à la généralisation des technologies numériques dont la fabrication additive, a donné naissance au mouvement des Makers et aux Fab-Labs ou ateliers de fabrication 3D ».

Sur cette base, certains pensent que ce ne seront plus les industriels qui fabriqueront les objets, mais les consommateurs eux-mêmes ou les Makers de proximité grâce à des fichiers 3D téléchargeables, fournis par l'industriel lui-même ou issus d'open sources sur Internet. Alors, un produit pourrait être l'égal d'un fichier. Si la réalisation de pièces en bonne matière est possible, la production (ou une partie de celle-ci) pourrait être relocalisée sur le sol national, permettant de gagner en frais de transport (limité alors à la transmission informatique d'un fichier). Il est donc important de regarder de près l'évolution de la technologie 3D qui peut faire basculer en partie les modes de production. Le tableau 3 inspiré de Deloitte (2014) rassemble des applications qui aujourd'hui sont sorties des laboratoires de recherche.

\begin{tabular}{|c|c|c|}
\hline & Avantages & Difficultés/remarques \\
\hline Prototypage rapide & $\begin{array}{l}\text { Coût modeste pour des pièces } \\
\text { complexes }\end{array}$ & $\begin{array}{l}\text { Qualité des objets réalisés avec des machines } \\
\text { domestiques }\end{array}$ \\
\hline $\begin{array}{c}\text { Délais } \\
\text { d'approvisionnement } \\
\text { et d'innovation }\end{array}$ & $\begin{array}{l}\text { A la main du concepteur par } \\
\text { réduction du nombre } \\
\text { d'intermédiaires ; } \\
\text { Procédés agiles }\end{array}$ & $\begin{array}{l}\text { Pièces uniques pour l'essentiel } \\
\text { Stockage de matière délocalisé }\end{array}$ \\
\hline $\begin{array}{l}\text { Diminution des coûts } \\
\text { indirects }\end{array}$ & $\begin{array}{l}\text { Peu de stocks et donc de frais } \\
\text { d'entreposage }\end{array}$ & $\begin{array}{l}\text { Matières parfois coûteuses } \\
\text { Recyclage des matières non utilisées possible }\end{array}$ \\
\hline $\begin{array}{l}\text { Personnalisation de } \\
\text { masse }\end{array}$ & $\begin{array}{l}\text { Possibilité d'usage unique ou } \\
\text { de quelques pièces adaptées à } \\
\text { un usage spécifique }\end{array}$ & Pas de fabrication collective \\
\hline Production de masse & Non sauf de petites séries & Oui, sauf de petites séries \\
\hline Matériaux & Large gamme & $\begin{array}{l}\text { Pas beaucoup de multi-matériaux - en } \\
\text { progrès }\end{array}$ \\
\hline $\begin{array}{l}\text { Aspects } \\
\text { environnementaux }\end{array}$ & $\begin{array}{l}\text { Moins de déchets } \\
\text { Technologie verte? }\end{array}$ & $\begin{array}{l}\text { Pas beaucoup d'études HSE (Hygiène, } \\
\text { Sécurité et environnement) }\end{array}$ \\
\hline
\end{tabular}

Tableau 3. Avantages et difficultés d'usage des procédés de fabrication additive

\subsection{La complexité est gratuite}

Cette technologie générique, permet par ses performances, la réalisation aujourd'hui de petites séries de pièces complexes inaccessibles aux méthodes classiques pour le même temps et le même coût (cf. figure 13 ; DIRECCTE, 2014 ; Pinkerton, 2016 ; Conner et al, 2014galement Weller, Kleer et Piller, 2015 ; Mellor, Hao et Zhang, 2014). De plus, en bout de chaîne informatique, ce procédé, sans (trop) d'intervention humaine directe, autorise une relation spécifique à l'objet (relation du même type que celle qui existe entre un citoyen et ses systèmes d'information et de communication). 


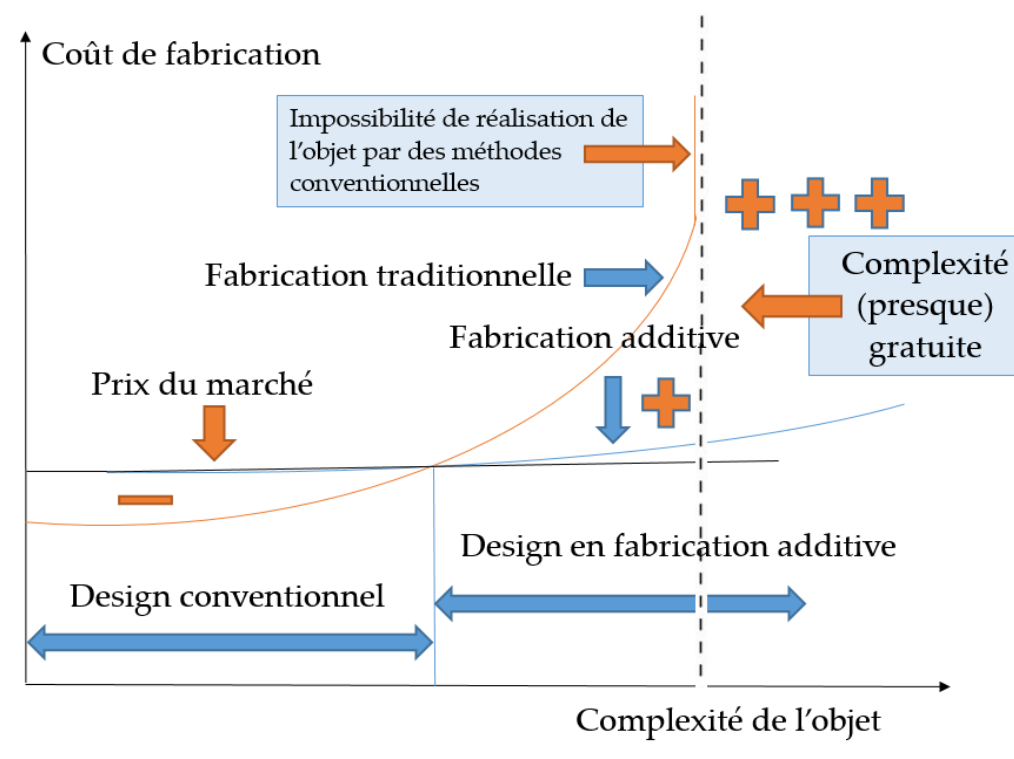

Figure 13. Intérêts et limites de l'impression 3D relativement à la conception d'un objet (le signe représente le domaine avantageux pour la conception traditionnelle, le + pour la conception en fabrication additive qui devient +++ quand l'objet est iréalisable par des méthodes conventionnelles)

Selon Dodziuk (2016), la pertinence (sans contestation) des technologies 3D se retrouve dans un certain nombre de situations (même en termes de développement durable) :

- Formes très complexes (exemple de buses d'injection, de pièces d'assemblage pour l'aviation avec moins de matériaux et un coût réduit de 75\%);

- Pièces mobiles comme des engrenages couplés ;

- Objets creux (exemple en aéronautique permettant un gain en poids transporté) ;

- Implants personnalisés et réalisation d'outils chirurgicaux personnalisés ;

- Prothèses ;

- Bio-printing (en devenir) ;

- Créativité et art ;

- Changement sociétal.

\subsection{Durabilité de la technologie}

GAO (2015) considère que fabrication additive peut s'inscrire dans les fondements du développement durable, en ce sens qu'il est possible d'éviter des pertes de matière. Cette donnée est d'autant plus vraie qu'une grande partie des matières non utilisées sont (au moins en partie) recyclables directement. Si cet aspect est non contestable, il faut cependant considérer que l'impression 3D rentre dans un processus global associant les technologies de l'information et de la communication (avec les matériels à durée de vie de quelques années qu'il faut produire et dont il faut gérer les déchets dangereux) et une consommation énergétique sous forme électrique et des coûts de matière qui peuvent être élevés. De plus, comme pour tous les autres procédés, il faut tenir compte des aspects environnementaux, d'hygiène et de sécurité.

Tang, Mak et Zhao (2016), dans un article de fond, en prenant comme fonction objectif l'analyse du cycle de vie, présentent les intérêts des re-conceptions en termes environnementaux sur quelques exemples d'objets réalisés en fabrication additive. Le tableau 4, issu de Reeves (2012), montre tout l'intérêt en termes de poids et de consommation d'équivalent carbone des technologies 3D. Cet avis est confirmé par Schwartz, Directeur des solutions innovantes de CENIT (CAO.fr, 2017) qui écrit : « Nous visons entre autres une réduction considérable du temps de développement complet des pièces d'impression 3D optimisées, ainsi que des économies significatives en termes de poids pour les avions, ce qui entraînera une réduction claire des émissions de $\mathrm{CO}_{2}$ sur l'ensemble de la durée de 
vie » (cf. figure 14). Ces données sont suffisamment significatives pour illustrer l'intérêt potentiel de la fabrication additive (cf. également, Ford et Despeisse, 2016). Elles sont encore à confirmer en tenant compte de tous les paramètres d'influence de la chaîne de production.
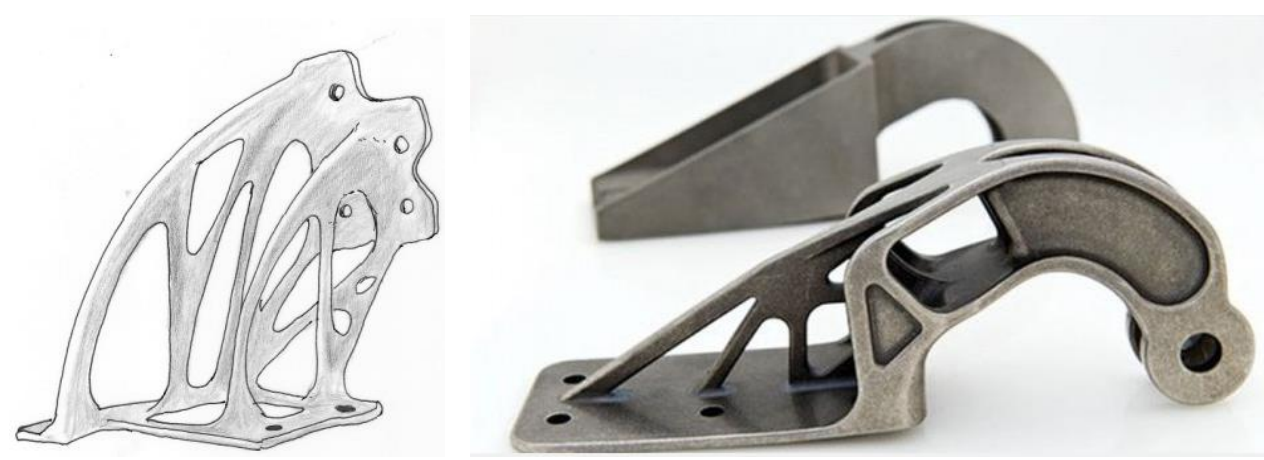

Figure 14. Dessin approché d'un redesign d'une pièce d'avion (fixation de siège) illustrant le gain en poids et comparaison entre fabrication traditionnelle et en fabrication additive (Alex, 2015 e)

«Aujourd'hui, les phases amont ont un impact majeur sur le coût des produits : $80 \%$ du coût final est fixé lorsque seulement 5\% des coûts de développements ont été engagés. Il devient donc crucial de mettre à disposition du concepteur des méthodes et outils qui lui permettent de prendre en compte les spécificités de la [Fabrication Additive] et qui s'intègrent au sein de l'éco système informationnel et humain déployé dans les entreprises : le Product Lifecycle Management (PLM) » (Garetti et al, 2005). Il faut donc repenser la conception parce que le procédé 3D le permet...

\begin{tabular}{|c|c|c|c|c|c|}
\hline $\begin{array}{c}\text { Procédé de } \\
\text { fabrication }\end{array}$ & $\begin{array}{c}\text { Matière }(\mathbf{k g} \\
\left.\mathbf{d e} \mathbf{C O}_{\mathbf{2}}\right)\end{array}$ & $\begin{array}{c}\text { Fabrication } \\
\left(\mathbf{k g} \mathbf{d e} \mathbf{C O}_{\mathbf{2}}\right)\end{array}$ & $\begin{array}{c}\text { Distribution } \\
\left(\mathbf{k g} \mathbf{d e} \mathbf{C O}_{\mathbf{2}}\right)\end{array}$ & $\begin{array}{c}\text { Usage } \mathbf{( k g} \text { de } \\
\left.\mathbf{C O}_{\mathbf{2}}\right)\end{array}$ & $\begin{array}{c}\text { Cycle de vie } \\
\left(\mathbf{k g} \mathbf{d e} \mathbf{C O}_{\mathbf{2}}\right)\end{array}$ \\
\hline Classique & 100 & 2 & 5 & 44 & 44 \\
\hline 3D avec treilllis & 16 & 5 & 1 & 16 & 16 \\
\hline 3D optimisé & 18 & 7 & 2 & 20 & 20 \\
\hline
\end{tabular}

Tableau 4. Avantage environnemental de la pièce redesignée

Les performances spatiales et mécaniques permises par la fabrication additive autorisent la mise au point de nouvelles voitures fortement allégées s'appuyant sur des bases biomimétiques (Casey, 2015), Selon Hart (2015), il est maintenant possible de réaliser des automobiles fonctionnelles par impression 3D, voitures qui peuvent rivaliser en termes de consommation, d'esthétique (mais pas encore tout à fait en termes de coût) avec les voitures réalisées selon des procédés classiques, etc. Mais aujourd'hui des éléments de véhicules, en petites séries, sont réalisés par impression 3D (3D Natives, 2018).

\section{Remarque : la question des pièces détachées}

Rufer (2014), à ce sujet, écrit : « Si on prend l'exemple d'un fabricant de machine à laver. Il doit en principe fournir des pièces de remplacement (souvent à des prix exorbitants) à tous les magasins qui vendent ses produits même si la plupart de ces pièces ne seront probablement jamais utilisées. Mais le commerçant est tenu d'avoir ces pièces en stock, lesquelles selon toute vraisemblance ont été fabriquées en Chine par exemple où à l'origine a été envoyée la matière première. De Chine, les pièces ont ensuite été dirigées vers l'entrepôt central qui les a réparties aux quatre coins de la planète. Bref, une simple pièce de rechange en plastique peut avoir fait deux fois le tour de la planète avant de se retrouver entre les mains des clients. D'un point de vue écologique, c'est inacceptable. D'autant plus que se développe aujourd'hui une prise de conscience collective des 
consommateurs qui intègrent largement l'impact écologique dans leur acte d'achat ». Le marché du remplacement de pièces défectueuses numérisées devient donc un marché économique profitable. Pour Tarit (2018) : "La pièce pourra être fabriquée localement sur commande avec un délai très court, diminuant ainsi l'impact carbone du transport. Les fabricants devront mettre les fichiers d'impression à disposition des clients, gratuits ou à titre onéreux ».

\section{L'impression 3D volumique}

L'idée d'André Franquin (1975) dans les « Petits formats » d'une photographie 3D commence à faire l'objet de publications, de brevets depuis peu. Plusieurs idées qui évitent de passer par l'étape d'additivité font l'objet de l'attention des chercheurs : absorptions multi-photoniques simultanées et séquentielles ; couplage transformation en polymère et son inhibition, etc. Ces technologies utilisent en général une seule source pour $\mathrm{n}$ voxels (l'objet) et évitent d'introduire des supports dans le processus de numérisation (cf. par exemple Amra et al, 2019; Shusteff et al, 2017; Kelly et al, 2019 ; Loterie, Delrot et Moser, 2019 ; Wang et al, 2019). La perte de temps éventuelle liée au processus multi-photonique est compensée par les gains en temps calcul et en élimination physique des supports. La figure 15 donne un exemple de pièce réalisée avec un de ces nouveaux processus de fabrication (Amra, André et Gallet-During, 2016).
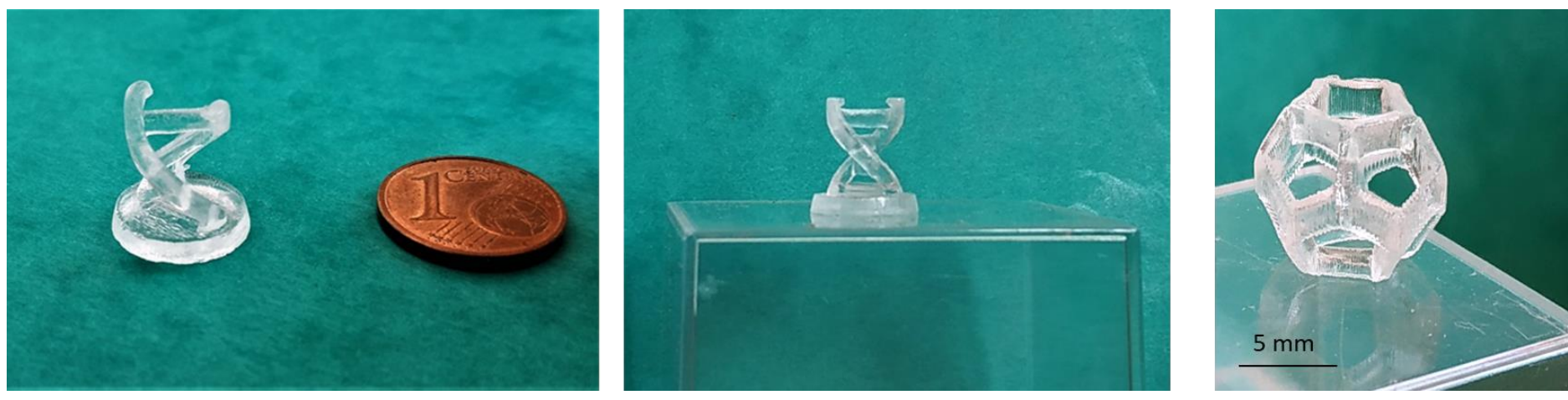

Figure 15. Exemple de pièces 3D en silice réalisée par absorption bi-photonique à partir d'un mélange résine acrylique et poudre de silice

\section{Du simple au compliqué, puis au complexe}

La question n'est alors plus ici de savoir comment les technologies 3D ont atteint leur légitimité, ni comment leur émergence a été permise par la Société, ni de décrire comment ces différentes technologies se sont imposées dans la compétition dans l'espace marchand. L'activité a impliqué l'exploitation d'un minimum de créativité, de savoir-faire et d'innovation incrémentale, de convictions traduites dans des preuves de concept. Ces résultats préliminaires répondent à certaines questions et permettent la l'émergence de nouvelles demandes, sur de nouvelles niches applicatives (cf. par exemple André, 2017).

La présente section concerne une transition vers de nouveaux horizons porteurs de futur: l'impression 4D et son volet biologique, le bio-printing, qui pourraient couvrir des marchés considérables (en milliers de milliards $€ /$ an), mais avec des technologies nécessitant des apports conceptuels nouveaux issus du domaine de la matière informée et/ou de la matière vivante.

\subsection{Impression 4D et bio-printing}

\subsubsection{Impression $4 D$}

L'idée sous-jacente est de partir d'une géométrie donnée pour qu'elle devienne par apport énergétique une forme correspondant à une application. En jouant sur la répartition spatiale et en 
amplitude d'une forme de cette énergie (de ces énergies), il y aurait également possibilité de disposer d'un système adaptatif comme un actionneur, d'un robot souple, d'une structure évoluant dans le temps et peut-être dans ses fonctionnalités (Zarek et al, 2017 ; Lehn, 2002 ; André, 2017). Ainsi, l'impression 4D issue des procédés de fabrication additive s'attaque à :

- La réalisation d'objets par impression 3D et dont la forme et/ou la fonctionnalité peut évoluer en fonction du temps ou de sollicitations extérieures ;

- L'assemblage d'objets ou de matériaux, qui s'assemblent par eux-mêmes, de par les propriétés de la matière qui les compose ou matière programmable. Des matériaux programmables qui s'auto-assemblent rendraient ainsi superflues les usines d'assemblages et les lourdes procédures d'installations. La robotisation, cœur des gains de productivité du XXème siècle, pourrait ainsi être intégrée aux produits eux-mêmes.

Si le volume de la littérature augmente de manière exponentielle depuis plus de 5 ans (quelques milliers de publication au total), le nombre de méthodes de déformation d'une macromolécule à cause d'une stimulation se limite à quelques principes classiques : chauffage, humidité, pH, phototransformation, électromagnétisme essentiellement. L'équipe de Nancy étant plus spécialisée en photochimie, photo-physique, le choix s'est tourné vers l'utilisation de la lumière comme stimulateur de déformation (André, 2018 ; 2018 a). De surcroît, ce principe basique permet d'exciter sélectivement certaines zones de l'objet $3 \mathrm{D}$ avec une grande précision et à distance. Cependant, plusieurs problèmes, non évoqués dans la plupart des publications sur le thème sont apparus (et sont pour l'essentiel valables pour d'autres modes de stimulation) :

\section{Tenue mécanique (E : module d'Young)}

Pour réaliser un objet actif en un seul matériau, il convient, à la fois, que celui ait une certaine tenue mécanique pour être un objet, et de propriétés élastomériques pour pouvoir se déformer. Avec des modules d'Young de quelques dizaines de MPa, classiques en impression 3D, les forces nécessaires à une déformation de $1 \mathrm{~mm}$ d'une barre de $1 \mathrm{~cm}$, épaisse de $0.1 \mathrm{~mm}$ et de largeur $1 \mathrm{~mm}$ sont de l'ordre de $2 \cdot 10^{-4}$ Newton. Les matériaux polymères classiques ne sont donc pas adaptés à une déformation pour réaliser un actionneur efficace ; d'ailleurs dans les publications, on montre juste des déformations, sans y associer un potentiel mécanique ou énergétique (cf. par exemple, Listek, 2019).

\section{Rendement quantique}

Si l'on veut provoquer grâce à la lumière la rotation d'une liaison double carbone-carbone ou azote-azote (cas de composés de la famille de l'azo-benzène), il faut absorber un photon d'énergie convenable. Mais ce processus se produit avec un rendement quantique parfois très inférieur à l'unité. Indépendamment de la possibilité d'absorption sur de petits éléments (loi d'absorption de Beer-Lambert), il faut qu'un grand nombre de liaisons soient activées, ce qui avec un rendement quantique de 0.5 par exemple peut conduire à une élévation très importante de la température locale. Par ailleurs, cela pose la question du choix de la puissance de la source lumineuse. De surcroit, dans le système chimique généralement retenu (qui doit être réversible) le composé Cis produit absorbe également (mais moins) que le composé le plus stable qui est le Trans (cf. figure 16). C'est le passage Trans-Cis qui induit la déformation qui est réversible par retour du Cis au Trans. 


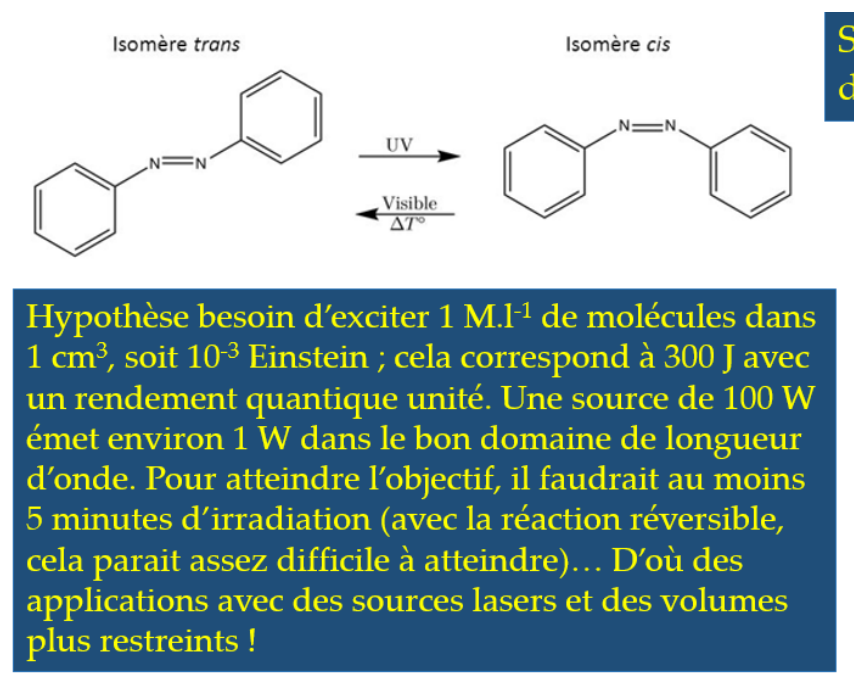

Absorption [u.a.]

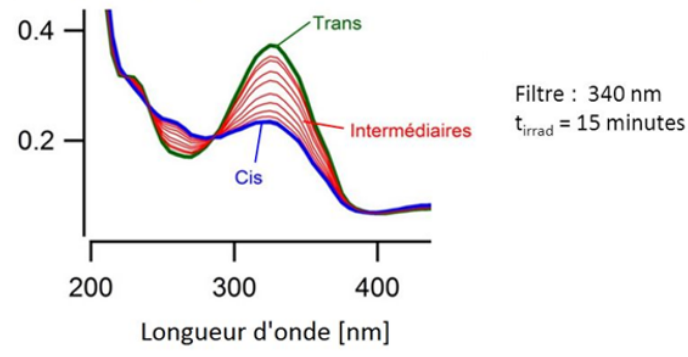

Figure 16. Problèmes associés à une stimulation par de la lumière

\section{Transfert de chaleur (Loi de Fourier)}

Les matériaux retenus dans la littérature sont essentiellement des polymères faiblement conducteurs de la chaleur. Si c'est la chaleur qui induit des transformations physiques de l'objet, il faut que la température change dans la masse du matériau (ce qui explique le choix au point 2- d'une stimulation par la lumière). D'un point de vue applicatif, le temps caractéristique moyen de transfert de chaleur sur $1 \mathrm{~cm}$ est de l'ordre de 15 minutes avec les polymères utilisés.

\section{Transfert de matière (Loi de Fick)}

Les transferts de matière sont généralement entre 10 et 100 fois moins rapides que ceux de chaleur. Les effets éventuels seront donc très lents. C'est ce qui se passe par exemple quand une déformation est induite par le $\mathrm{pH}$, nécessitant la diffusion d'atomes $\mathrm{H}+$ dans le matériau massif.

\section{Anisotropie de la fabrication}

Si l'on réalise un objet par stéréo-lithographie (pour l'instant à 1 photon), la lumière arrivant sur la surface à polymériser est plus absorbée à la surface qu'en profondeur. La partie présente vers la surface est donc plus polymérisée (voire rigidifiée) que les zones situées plus en profondeur. Avec des monomères synthétisés au laboratoire, nous n'avons jamais été capables de trouver les bonnes conditions d'élasticité et/ou de tenue mécanique prévues. De surcroît, l'absorption conjointe de la lumière par la composante azo-benzène de la résine et par l'amorceur se traduit par une polymérisation de plus en plus complète, avec un durcissement massif de l'objet.

\section{Autres problèmes}

Ne sont pas évoqués ici d'autres problèmes qui seraient à prendre en considération : celui de la maîtrise mathématique des déformations, du design optimal, ni de l'encombrement du système de production de stimuli.

\subsection{2. bio-printing}

Le morphing ou matière programmable s'introduit en anticipation scientifique et technologique dans de nombreuses tendances de développement. Alors, pourquoi ne pas évoquer une transformation spatiale et fonctionnelle d'un objet contenant des cellules vivantes par l'apport spécifique d'énergie, quelle que soit sa forme et sa nature? 
Avec l'individualisme, la spécificité des corps risque de devenir l'un des fondements de la médecine de précision de demain, en tant qu'elle permet des soins optimisés (et moins coûteux en principe). Dans le même temps, les effets de la transformation du concept de santé (OMS) allant de la suppression de la maladie, à la réalisation d'un bien-être individuel (médecine de confort) soutiennent le développement potentiel d' "Human Enhancement », développement autorisé dans son principe par le bio-printing. De plus, l'avènement de l'impression 3D pourrait résoudre certains des problèmes les plus pressants auxquels fait face le don d'organes - comme la disponibilité et le risque de rejet par le corps du patient. Au lieu d'utiliser de l'encre, ces imprimantes d'organes peuvent déposer un mélange de cellules vivantes (appelées encre-bio ou bio-ink), couche par couche, pour former des tissus humains. Comme les organes sont imprimés avec les cellules du patient, elles ne seront en principe pas rejetées en cas de transplantation. Le succès de l'impression d'organes humains utilisables semble donc prometteur et fortement désirable...

Le bio-printing est une technique de fabrication additive permettant d'imprimer en trois dimensions des couches successives de cellules sur des bio-matrices afin de reconstituer à l'identique la structure d'un tissu, voire d'un organe entier. Il est imaginable qu'à partir de concepts basiques on s'élèvera progressivement d'essais de laboratoire à des réparations de tissus biologiques (Parrochia, 1994). Le bio-printing appartient à l'ingénierie du vivant ou à la bio-ingénierie, qui intègre les sciences physiques, chimiques, mathématiques, ainsi que les principes d'ingénierie pour étudier la biologie, la médecine, les comportements et la santé pour la fabrication d'organes vivants. Il constitue une forme de chaînon manquant entre l'artificiel et le naturel.

Pour réaliser un bio-construit, il est possible de partir d'une forme donnée, facile à réaliser, pour qu'elle devienne, par apport de nutriments et grâce à une composition adaptée (énergie chimique par exemple), une forme correspondant à une application. En jouant sur la répartition spatiale et en amplitude de cette énergie (de ces énergies), il y aurait également possibilité de disposer d'un système adaptatif comme un élément d'organe vivant. Sans doute dans ces processus cherche-t-on à appliquer des formes de déterminisme qui feraient l'hypothèse que tout est donné avec la connaissance des seules conditions initiales...

Sur ces bases, la vision initiale de l'ingénieur est de faire simple, si possible à l'intérieur d'une pensée causaliste, avec les outils à sa disposition et de rejeter, pour autant que le résultat soit obtenu, ces concepts disruptifs et les non-linéarités qui sortent trop de sa culture. La matière vivante peutêtre ordonnée, signifiant que sa forme peut-être, au moins en première approximation, décrite géométriquement. On peut aussi penser que les structures, plus ou moins auto-organisées, doivent être associées à certains types de conditions physiques ou physico-chimiques. Mais, en se plaçant dans une perspective évolutionnaire plus large, il n'est pas possible de prédire l'évolution des formes en les déduisant de l'application des lois simples qu'il aurait pu identifier. Plus exactement, à l'expérience, si ces lois sont nécessaires, elles ne sont pas suffisantes. Pour autant, de nombreux travaux concernent la réalisation d'amas cellulaires qui, disposant de propriétés d'auto-organisation peuvent constituer des tissus plus ou moins viables (quelques semaines). La réalisation d'organes reste encore utopique car il n'y a pas eu encore convergence des disciplines concernées pour permettre la maitrise des phénomènes biologiques dans les bio-construits (cf. par exemple, André, 2017).

La figure 17 présente le principe général retenu pour une fabrication par bio-printing à partir de ses propres cellules (prises en nombre minimal sur la personne). 


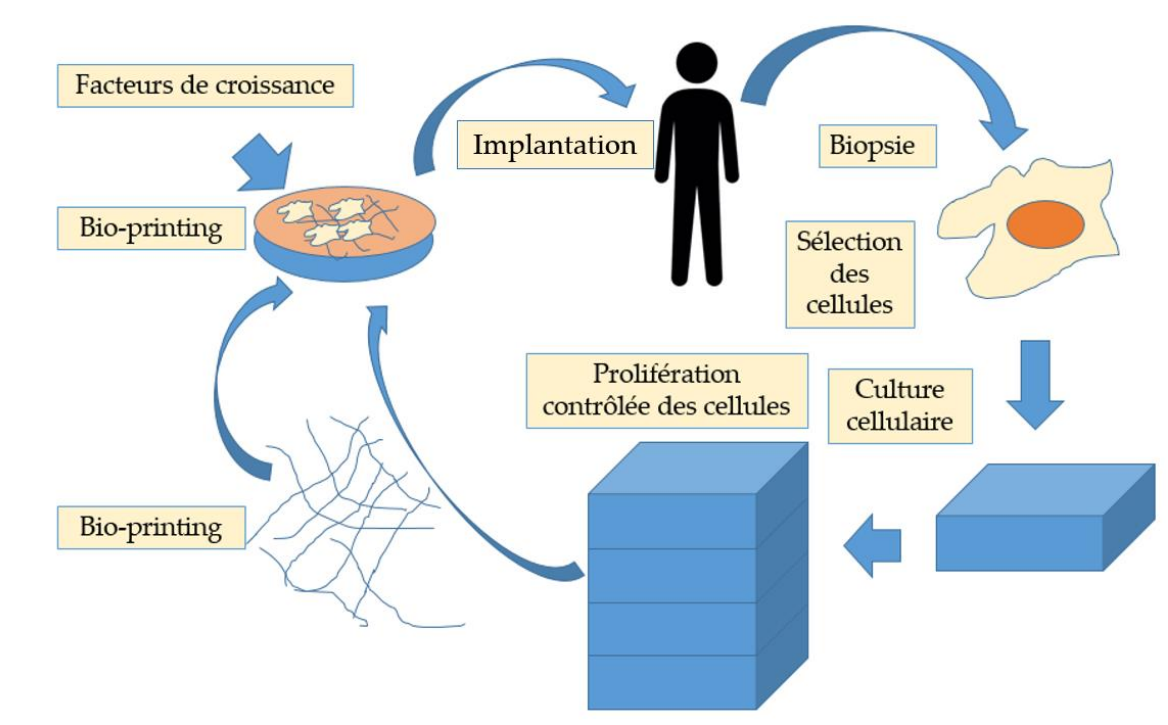

Figure 17. Principe général d'utilisation (cercle vertueux) du bio-printing

L'impression d'éléments vivants de toutes natures relève alors d'une technique classique de fabrication additive appliquée à de la matière vivante (avec les conditions de propreté requises). Mais, ce n'est pas trop la technologie qui est problématique, c'est son utilisation avec des cellules vivantes, qui doivent le rester dans le processus de fabrication et qui explore la complexité des phénomènes de la Nature...En effet, plusieurs problématiques doivent être maîtrisées (Guédon, Malaquin et André, 2017).

\section{Richesse cellulaire}

Une question initiale à ce stade est de savoir si la richesse cellulaire doit être introduite dans la construction d'un tissu par bio-printing (ce qui parait pour le moins délicat avec les machines actuelles), ou si l'on se satisfait d'un lot réduit d'espèces cellulaires qui auront pour mission de proliférer et de se différencier ultérieurement pour atteindre l'objectif prévu par le chercheur. Le niveau actuel concerne l'utilisation de cellules souches indifférenciées.

\section{Maturation}

Les cellules extraites en faible quantité doivent se développer préalablement dans un milieu de culture adapté. La question posée est de disposer à temps d'un stock suffisant de cellules pour la bio-fabrication.

\section{Support de cellules}

D'un point de vue technologique, le dépôt de cellules ne permet pas leur survie par manque d'un flux de nutriments et d'oxygène suffisant. Une seconde question émerge alors, celle liée à la présence d'échafaudages (scaffolds) créés en fabrication additive sur lesquels les cellules vont être déposées, question qui nécessite pour une réponse acceptable un choix adapté de matériaux et un design adapté pour que les cellules déposées se développent... en espérant que ces supports biodégradables disparaitront à temps pour que le tissu ressemble à celui qu'il doit remplacer.

\section{Différentiation cellulaire}

L'idée générique du bio-printing est représentée sur la figure 18: placer sur un support les cellules, sur un échafaudage pour qu'un tissu vivant puisse se développer (Melchels et al, 2012). Au nom du bio-printing, des chercheurs s'appliquent à construire le plus intelligemment possible des briques de vivant, voxels complexes et à la fois très simplifiés et contrôlables, qu'il suffirait ensuite 
d'empiler pour obtenir des tissus viables répondant à un cahier des charges fonctionnel. Sur la figure 19, le développement cellulaire s'opérationnalise autour d'un flux de matière et d'énergie.

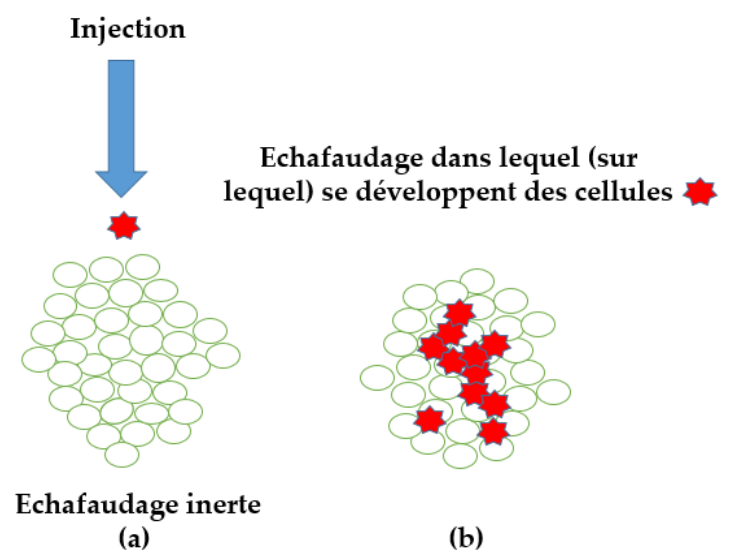

Figure 18. Association support - dépôt de cellules dans le bio-printing

(a)

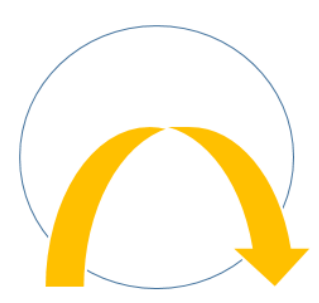

(b)

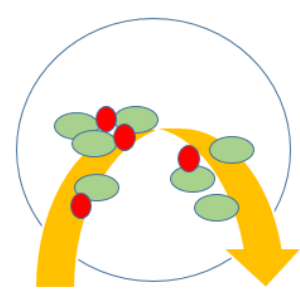

(c)

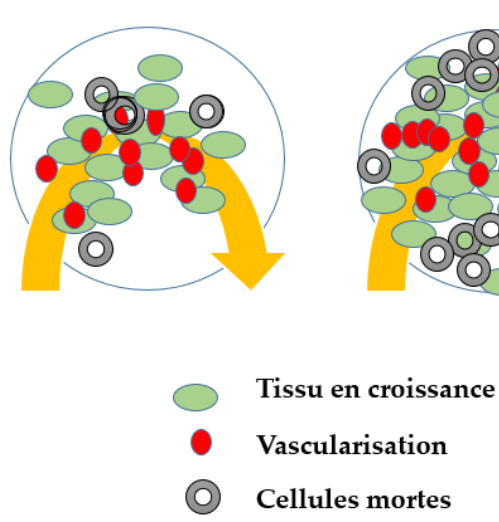

(d)

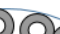

(1)

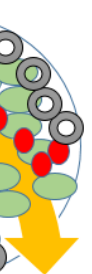

Figure 19. Principe du bio-printing avec des changements dans le temps des matériaux vivants imprimés

(André, 2017)

\section{Bio-fabrication}

Cette étape nécessite des précautions spécifiques surmontables ; elle ne constitue cependant pas la fin de l'histoire car il faut que le tissu cellulaire se développe et se différencie comme prévu. Selon Jessus et al (2017), « la morphogénèse [...] est un processus d'auto-organisation de cellules qui grandissent, se divisent, se déplacent, ou meurent en fonction des événements auxquelles elles ont été soumises dans le passé, de leur histoire familiale et des communications qu'elles établissent avec leurs voisines »... Bref, on est bien loin de la fabrication additive traditionnelle... Pour enfoncer le clou, elle écrit également : «Certaines cellules animales cultivées sur des gels adoptent des destins différents selon la rigidité mécanique du gel : elles deviennent des cellules de cerveau sur un gel mou, d'os sur un gel dur ou de muscle sur un gel de rigidité intermédiaire. [...] En réponse aux forces qui leur sont appliquées, les cellules réorganisent leur squelette interne pour mieux résister à ces forces, pour changer de forme ou pour orienter leur prochaine division $\gg . .$.

\section{Implantation}

Après sa phase de maturation le bio-construit doit être intégré en lieu et place du tissu ou de l'organe à remplacer, ce qui fait l'hypothèse qu'on peut raccorder facilement nerfs, vaisseaux 
sanguins au milieu biologique conservé, en faisant l'hypothèse que le bio-construit aura la bonne fonctionnalité et les bonnes taille et forme...

\subsection{Pour aller plus loin dans le domaine 3D}

Ce que l'on constate également c'est la difficulté de la recherche d'explorer des domaines plus compliqués comme l'utilisation de voxels de tailles variables, de travailler avec plusieurs matériaux, d'anticiper des aspects de type problème inverse de manière robuste grâce à des simulations tenant compte des comportements des matériaux dans les procédés, etc. Dans le domaine des technologies $3 \mathrm{D}$, la construction des connaissances dispose encore d'un avantage tactique qui manque généralement à l'étude de la maîtrise des phénomènes naturels (ou simplement complexes). En effet, nous savons comment les artéfacts ont été conçus depuis 30 ans environ avec des hypothèses très plausibles, proches des connaissances disciplinaires permettant la réalisation de cahier des charges pour la réalisation de maquettes instrumentales sur lesquelles on peut expérimenter et valider des améliorations incrémentales. C'est sans doute ici que le cadre exploratoire de la fabrication additive a pris et continue à prendre toute sa place en étant producteur d'intelligibilité scientifique et d'applications... Mais déjà, en partant du causal disciplinaire, on aboutit de manière insidieuse à du compliqué pluridisciplinaire, voire plus.

\subsubsection{Complexité}

« L’image classique du monde était bien décrite par Feynman (1965) [...] quand il comparait la nature à une immense partie d'échecs. Chaque mouvement, pris isolément, serait simple et la complexité tout comme l'irréversibilité résulteraient simplement du grand nombre d'éléments en jeu. Mais, aujourd'hui il est difficile d'accepter cette image... » (Prigogine, 1994). Nous voici donc dans la complexité et la mutualisation interdisciplinaire... Le bricolage savant des années 1980 qui a fait ses preuves s'est transformé en ajoutant des éléments contributifs incrémentaux comme l'amélioration des procédés, des matériaux, des logiciels, etc. C'est ce qui conduit au succès actuel des technologies de fabrication additive, parce que chaque élément ajouté a constitué un progrès . Aussi, il n'a pas perturbé de manière notable les dispositifs précédents (ni le concept de base). Mais, en intégrant de manière plus complète des éléments issus de corps de savoirs disjoints, les acteurs sont confrontés à des questionnements nouveaux liés à des connaissances insuffisantes sur les interactions entre les éléments, à l'accès à ces connaissances. Dans le cas du bio-printing, la maîtrise des mécanismes qui gouvernent par exemple la croissance cellulaire, sa différenciation, etc. est imparfaite.

Ce n'est pas tant la multiplicité des composants, ni même la diversité de leurs interrelations, qui caractérisent la complexité d'un système : tant qu'ils sont pratiquement et exhaustivement dénombrables, on est en présence d'un système compliqué, dont un dénombrement combinatoire pourrait (si on a le temps et les moyens) permettre de décrire tous les comportements possibles (et par là de prédire son comportement effectif à chaque instant dès que la règle ou le programme qui les régit est connu) : en termes mathématiques on est en présence d'un problème polynomial. C'est l'imprévisibilité potentielle (non calculable a priori) des comportements de ce système, liée en particulier à la récursivité qui affecte le fonctionnement de ses composants : en fonctionnant, ils se transforment, suscitant des phénomènes d'émergence, certes parfois intelligibles, mais non toujours prévisibles. Les comportements observés des systèmes vivants fournissent d'innombrables exemples de cette complexité, et le bio-printing en fait partie (cf. Jessus, 2017 ; André, 2017). Avec des matériaux vivants, ce que montre Jessus (2017), c'est que le devenir d'un bio-construit est sensible aux conditions initiales (qui ne sont pas toutes connues); c'est une autre forme de complexité qui est à l'œuvre.

Avec le bio-printing, la vision traditionnelle est de rechercher si une rigueur mathématique, des procédures algorithmiques peuvent assurer à l'interface entre technologie, chimie, sciences de l'ingénieur et biologie, un corps de doctrine conceptuel pour la production de tissus et d'organes 
biologiques. Dans un esprit d'étayage empirique maximal (rasoir d'Occam), l'idée mobilisatrice est de fonder un modèle théorique/empirique robuste dont le surplus de contenu descriptif est minimal par rapport à ce qui est indispensable pour rendre compte des phénomènes. On caractérise ainsi cette vision réductrice comme un travail d'ingénierie, travail encore en devenir. Mais, en fait, dans ce qu'écrit Jessus (2017), on retrouve ici une information qui s'apparente à la sensibilité aux conditions initiales évoquée plus haut.

Pour faire sauter ces (grandes) difficultés, plusieurs disciplines devraient être à l'œuvre, ce qui amène à modéliser plusieurs domaines ayant probablement des équations de comportement différents, des interfaces multiples et singulières, des non-linéarités, des changements d'échelles (depuis les composants subcellulaires jusqu'à l'organe) associés à des formes fractales éventuelles, etc. La complexité envahit le domaine par plusieurs voies : celle des arrangements géométriques, de la complexité de la dynamique (croissance, retrait de l'échafaudage provisoire par exemple) et celle de la fonctionnalité, de la compétition entre systèmes cellulaires, de la reproduction,... (André et Larger, 2016; Gleick, 2008). Une question associée concerne pour une action déterminée, l'effet d'un stimulus sur cette dernière, avec une possible comparaison entre l'amplitude du stimulus d'entrée et son effet (Cunchillos, 2014) avec des aspects, au moins apparemment, contingents, spontanés et intentionnels (Mais, comment la lecture du génome permet-elle de prédire le futur du système biologique ?) (Pictet, 2015)).

C'est vrai que sur des sujets ouverts, le débat entre les tenants du bottom-up et ceux qui envisagent préalablement à toute action une réflexion intégrative risque d'apparaître dans une approche heuristique stabilisée comme une discussion sans fondements robustes par impossibilité de trancher et d'avancer. Les premiers - qui, pionniers d'un domaine émergent - utilisent des méthodes d'essais et d'erreurs pour tenter de réaliser des preuves de concept et être parmi les premiers à publier sont soumis à la pression de la compétition. Les seconds, plus rares, envisagent préalablement à toute action une réflexion intégrative (top-down) de type slow science. Qui croire entre ceux qui promettent et les freineurs qui, comme Plick et Plock (Christophe, 1985), veulent réfléchir avant d'agir? Ces derniers, souhaitent trouver du sens à une approche heuristique définie sur la figure 20 ; par leurs modélisations, leur volonté de cotraitance. Ils envisagent d'être des passeurs de frontières. Débattre, c'est cependant tenter de sortir de promesses difficiles à tenir, de certains stéréotypes paralysants, de désirabilités applicatives, de fausses promesses, de compétitions, d'émotions et de recherche de reconnaissance, voire simplement de foires d'empoigne.

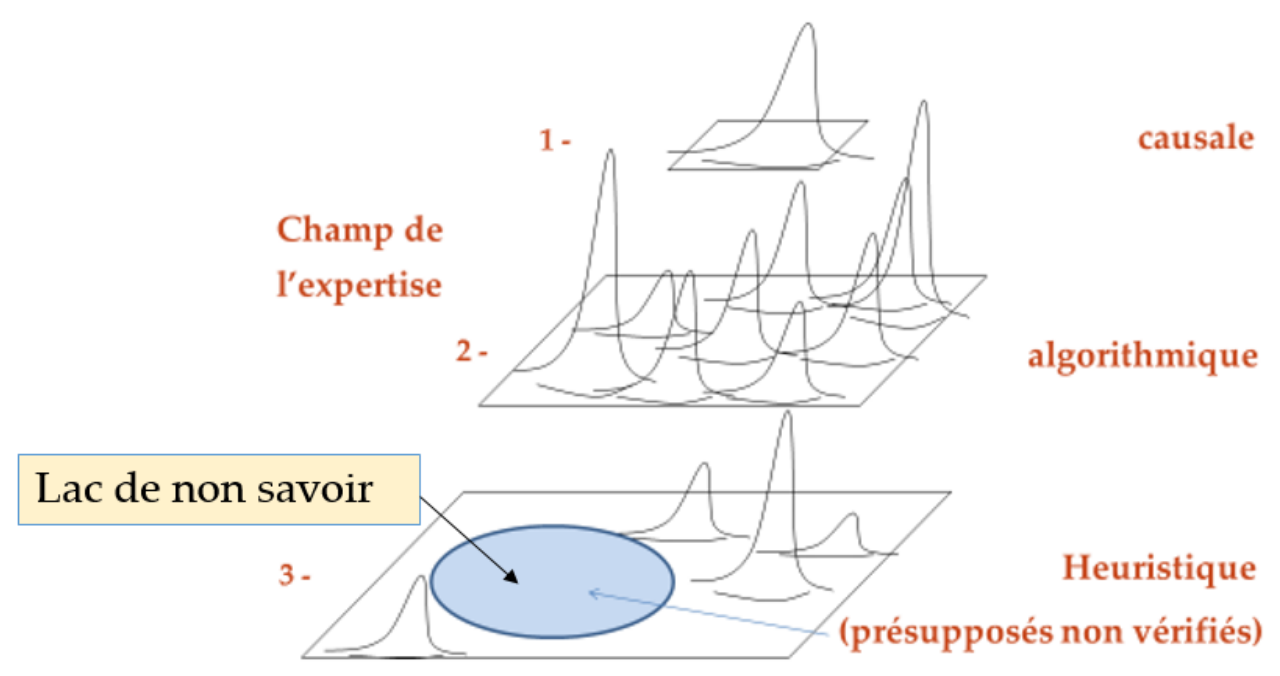

ELARGISSEMENT DU CHAMP DE L'EXPERTISE

Figure 20. Champs d'expertise. Les gaussiennes approximatives représentent les acquis scientifiques issus du travail de recherche antérieur ; selon les formes d'expertise, le domaine est couvert (causal, algorithmique) ou partiellement couvert (heuristique) 
De surcroît, il faut rappeler que l'on travaille toujours sur un corpus de connaissances incomplètes dont le degré de généralisation à des échelles plus grandes ou plus petites peut/doit être remis en cause. L'idée cependant est d'étudier les paramètres de contrôle - ceux qui sont connus (température, flux divers, hétérogénéités diverses, etc.) - pour tenter d'examiner l'existence d'attracteurs et leur(s) déformation(s) induite(s) par l'environnement en se rapprochant des conditions réelles avec des dispositifs adéquats. En effet, la plupart des processus responsables du fonctionnement du vivant, à toutes échelles - de la cellule à l'écosystème - résultent des interactions de dizaines, de centaines, voire de milliers de facteurs : présence ou absence d'un ion, d'une molécule, d'un nutriment, d'un gène, conformation d'une protéine, etc. Approfondir leur compréhension suppose donc de maîtriser des outils susceptibles de représenter et d'analyser ces interactions complexes. C'est ce que l'on désigne aujourd'hui sous les termes de biologie des réseaux (Vandeginste, 2012), à moins que la Nature fasse bien les choses, ce qui est possible.

Les lois de croissance biologiques sont associées à des phénomènes dynamiques non linéaires du fait de la richesse des nombreux scénarios déjà observés. L'appropriation du domaine dans une approche transversale (impliquant toutes les disciplines concernées) est de manière évidente un élément clé pour faire avancer le sujet. La culture des Systèmes Dynamiques Non Linéaires (SDNL), certes complexe, est encore peu diffusée. Rappelons que dans les systèmes linéaires, les modifications des paramètres entraînent des changements quantitatifs, mais ne modifient pas le comportement (état stationnaire) du système. Dans les SDNL, une petite variation de certains paramètres, dits paramètres de contrôle, peut dans des conditions bien définies, au voisinage d'une valeur critique, provoquer un changement complet de comportement à l'équilibre du système. C'est une bifurcation, et celles-ci apparaissent souvent dans les milieux biologiques.

\subsubsection{Notion de convergence}

Scientifiquement, l'approche par la complexité est une rupture qui reconnaît le caractère exceptionnel et problématique du vivant (hard et soft). Il peut être ici utile d'invoquer dans ce cadre, la nécessaire acceptation de l'incertitude et de la démarche heuristique dans la compréhension des problèmes dits complexes reliés par exemple à la santé, au comportement d'une seule personne (et non d'une population), donnant ainsi une valeur plus grande au qualitatif, au sensible, à l'heuristique, plutôt qu'au seul quantitatif (André, 2017 a).

Il faut donc envisager que les fondements épistémologiques de la fabrication additive du futur s'appuient de plus en plus sur le paradigme de la complexité, où l'interdisciplinarité est envisagée comme un des moyens d'étude. L'approche disciplinaire est trop souvent cloisonnée, d'où une idée maîtresse visant à savoir comment percoler au travers des frontières disciplinaires afin que le paradigme de la complexité puisse se déployer véritablement. Ainsi, la recomposition de catégories de pensée ne reposerait plus sur des frontières et des objets disciplinaires, mais sur des objetsfrontières portés par des créatifs, des divergents, qui n'ayant pas peur des récursivités, souhaitent répondre légitimement aux grands enjeux auxquels la société doit faire face. Ce changement de portage de la recherche vise une réelle intégration responsable d'activités ouvertes vers la société, porteuses de sens. C'est une voie permettant de faire émerger de l'indiscipline en fabrication additive comme un des acteurs scientifiques crédibles et opérationnels des mouvements technoscientifiques et sociétaux actuels. C'est peut-être un moyen de contribuer à l'usine du futur avec des critères revisités, moyen qui n'émerge qu'imparfaitement dans les faits présents. L'exemple du bio-printing, applicable également à des degrés différents au 4D Printing, illustre le propos visant la convergence de nombreuses disciplines comme l'illustre la figure 21 . 


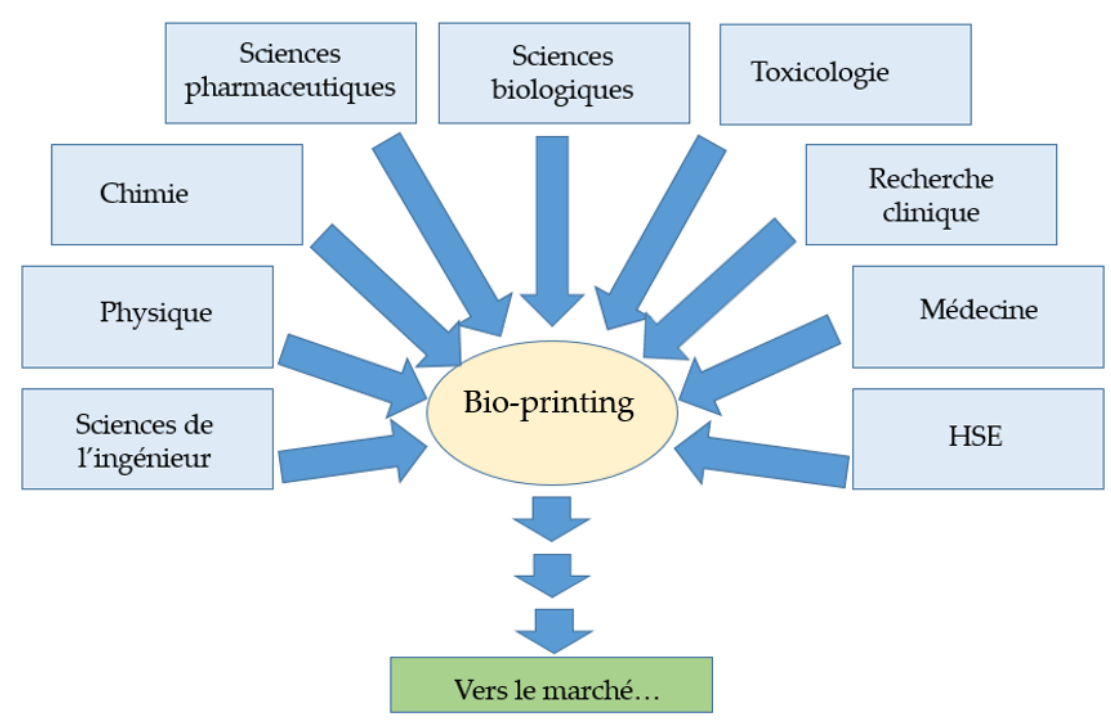

Figure 21. Convergence et Bio-printing (HSE : Hygiène, sécurité et environnement)

\section{Une conclusion provisoire}

Sur la base d'une analyse plus approfondie, des axes de redéploiement devraient être proposés permettant d'engager des évolutions culturelles plus responsables, scientifiques et des organisations plus flexibles adaptées aux nouveaux buts poursuivis, car porteurs d'applications utiles pour la société. C'est à l'aune de transformations de fond et de la prise de risques que l'on saura si la fabrication additive restera à même d'être un acteur efficient entre la recherche disciplinaire et la société de production. Plusieurs pistes d'évolution des systèmes de production sont aujourd'hui avancées dans de nombreux rapports : changements sociétaux, personnalisation «de masse », économie circulaire, servicisation et économie de fonctionnalité, territorialisation de la production et des savoirs, économie de la connaissance, développement de nouvelles compétences, communautés d'acteurs à forte interopérabilité, innovation intégrée dans un processus continu essentiel au positionnement concurrentiel de la recherche, des entreprises et à leurs performances...

L'objectif de toute modélisation est pour tout professionnel un peu technicien de fournir un système paramétrable quand c'est possible. Il s'agit bien de l'optimiser relativement à une fin, grâce à un choix judicieux de paramètres d'entrée et un asservissement stable et robuste dans sa dynamique. L'environnement des systèmes du bio-printing (voire de l'impression 4D) nécessite la connaissance robuste des variables indépendantes et interdépendantes qui conditionnent son état à un moment donné. Plus ce système est complexe, plus il faut classiquement le décrire à l'aide d'un nombre d'équations couplées, en nombre suffisant. Pour l'ingénieur, cette description doit exploiter le nombre le plus restreint possible de paramètres, compte tenu de ce qui est mesurable, mesuré et utile à la finalité. Pour lui, le modèle que l'on souhaite élaborer se situe généralement entre efficacité et vérité, où il est souhaité un compromis entre fidélité à la réalité et simplicité de mise en œuvre (principe d'optimisation dans un cadre de robustesse donné). Mais quelles sont les approximations susceptibles de rendre le modèle opérationnel selon un cahier des charges imposé par les utilisateurs ou par le corps médical ? Comment la technologie peut-elle rapprocher les points de vue et lever (toutes) les contraintes et les injonctions paradoxales ? Pour atteindre un tel objectif, il faut croiser les savoirs des différentes disciplines et métiers convoqués, avec les difficultés associées, mais sera-ce suffisant?

En proposant une démarche interdisciplinaire et inter-métiers, le projet n'aspire pas à faire jouer à l'approche un autre rôle que celui d'être au service de chaque discipline et à chaque savoir technique. En se donnant pour but de mettre en évidence, par exemple, que la démarche complexe conduit à la formulation d'une hypothèse explicative ou interprétative, peut permettre d'instaurer un rapport de dialogue et de mutualité entre partenaires. Précisons que le projet ainsi esquissé 
présuppose l'adhésion au point de vue de l'ignorance métaphysique... Dans cette optique, le projet devrait avoir pour rôle d'impulser une réflexion critique sur les idées directrices, les intentions fondatrices, les concepts directeurs, les présupposés méthodologiques, paradigmatiques, anthropologiques, épistémologiques, éthiques de chaque discipline et des connaissances du champ des possibles de chaque métier. Mais en même temps il doit accepter de se cantonner humblement dans une tâche de clarification, de débroussaillage le plus savant possible, qui ne saurait a priori prétendre à une clarté ultime ou à une vérité première et fondatrice.

Voilà donc l'objet qui vise un vrai soutien à l'exploration de la complexité pour le redéploiement de la fabrication additive sur de nouvelles niches. En premier lieu, il s'agit déjà de sortir des simples preuves de concept en vérifiant la reproductibilité des expérimentations (ce qui est parfois fastidieux en bio-printing, mais indispensable). En second lieu, il s'agit bien d'investir les aspects convergence interdisciplinaires et inter-métiers technologiques dont chacun reconnait la difficulté et la faible reconnaissance par la hiérarchie. La modélisation du passage en 4D printing d'une sphère à un cube (et réciproquement) est encore à espérer - De là à construire des machines opérationnelles !

Présentée comme domaines éminemment collaboratifs, l'impression 4D et le bio-printing, de par la nécessité d'intégrer des principes de convergences scientifique et technologique, offrent un terrain d'observation particulièrement intéressant de l'interdisciplinarité orientée vers un but applicatif réel avec des développements économiques possiblement considérables. Sur cette base, des alliances disciplinaires et inter-métiers doivent, après apprentissage d'un langage de communication commun, permettre de réfléchir aux meilleures façons d'explorer ces domaines porteurs sans qu'on puisse aujourd'hui savoir si l'on saura traiter le problème inverse ou définir une relation causale entre projet d'application et fonctionnalités attendues. De fait, la consultation de différents partenaires issus de disciplines et milieux différents doit s'exprimer à travers une diversité de courants révélatrice de la dimension, de la complexité et des tensions des domaines émergents de la fabrication additive. Il en résulte normalement des difficultés de coopération entre des acteurs fortement hétérogènes, mais c'est peut-être surmontable si l'on s'en donne les moyens, permettant d'examiner si les difficultés sont ailleurs. Ce développement obligé doit intégrer une thermodynamique complexe avec une entropie forte qui doit être acceptée par le corps de la recherche dans son ensemble.

Mais qui acceptera aujourd'hui dans le fonctionnement de la recherche académique de prendre le risque d'échecs?

\section{Bibliographie}

- 3D Natives (2018) «BMW accélère l'intégration de la fabrication additive dans son cycle de production» https://www.3dnatives.com/bmw-fabrication-additive-170420183/

- Adamson A.W. (6 Dec. 1968) "Method and apparatus for generating 3 dimensional patterns" US Patent 3609706 A.

- Adelman A.H., Lewis J.D. (6 Dec. 1968) "Method and apparatus for generating 3 dimensional patterns" US Patent 3609707 A.

- AFNOR (2011) « «NF E 67-001» Octobre 2011 : Fabrication additive - Vocabulaire » AFNOR Ed. - Paris.

- Amra C., André J.C., Gallais-During L. (2016) « Procédé pour la réalisation d'un objet tridimensionnel par un processus de photo-polymérisation multi-photonique et dispositif associé » Brevet français 16-59211 du 28-092016. Dépôt d'une demande internationale de brevet (PCT) concernant l'invention citée en référence : numéro PCT/FR2017/052622 du 27-09-2017.

- Amra C., André J.C., Gallais-During L., Zerrad M. (2019) « Procédé de fabrication d'un objet tridimensionnel, ou de modification de l'état de surface d'un objet préformé, par photo-polymérisation » Brevet français FR 1908646 du 30-07-2019.

- André J.C. (2016) « Désordres dans la troisième dimension ; un cheminement entre formation et interdisciplinarité » VARIA, $\underline{5}, 62-72$. 
- André J.C. et Larger L. (2016) «Approche épistémologique du bio-printing pour essayer de moins mutiler la recherche sur un objet-frontière » Rapport au CSI-INSIS - CNRS - Paris.

- André J.C. (2017) "From Additive Manufacturing to 3D/4D Printing" - Volume 1: "From the first concept to the present applications"; Volume 2: "Improvement of the present technologies and constraints"; Volume 3: "Breakdown innovations: Programmable matter; 4D Printing and Bio-Printing" ISTE/Wiley Ed. - Londres - UK.

- André J.C. (2017 a) « Convergence NBIC : risques et conditions de possibilité au regard d'autres risques silencieux » Environnement Risques et Sante, 16, 178-191.

- André J.C. (2018) "From self-organization to 4D (Bio)-Printing - A plea to go beyond exciting proof of concept" Conférence invitée "Von Karman Conference" - Cologne - Allemagne 15-18 Juillet 2018.

- André J.C. (2018 a) « De l'auto-organisation à l'impression 4D» Industries et technologies, 1012, 57-63.

- André J.C. (2019) “Industry 4.0 - Paradoxes and Conflicts” ISTE/Wiley Ed. Londres - UK.

- André J.C., Le Méhauté A., de Witte O. (1984) «Dispositif pour réaliser un modèle de pièce industrielle » Brevet français n ${ }^{\circ} 8411241$ du 16.07.1984 (suivi de plus de 25 autres)..

- Andrzejewska E. (2001) "Photo-polymerization kinetics of multifunctional monomers" Progress in Polymer Science, 26, 605-665.

- Bhushan B., Caspers M. (2017) "An overview of additive manufacturing (3D printing) for microfabrication" Microsyst. Technol., 23, 1117-1124.

- Bouffaron P. (2014) «Impression 3D : Les prémisses d'une nouvelle (r)évolution industrielle » ambassade de France aux Etats-Unis 48 pp. http://sf.france-science.org/wp-content/uploads/2014/11/SMM14 025.pdf

- Branche P.M. (2015) « Impression 3D ; la fin annoncée des transports » http://www.voxlog.fr/dossier/13 2/logistique-et-transport-limpression-3d-remet-en-cause-la-supply-chain

- Brethome K., Palacin B., Puech J., Carpentier L. (2016) «Les technologies additives pour les applications spatiales » Workshop INSIS CNRS « Fabrication additive pour l'électronique » - Paris http://www.cnrs.fr/insis/recherche/docs-evenements/workshop-INSIS 11.01.16 LCarpentier.pdf

- CAO.fr (2017) «CENIT participe au projet de recherche européen «BionicAircraft » » http://www.cao.fr/rubriquetoutes/actu/7311/CENIT-participe-au-projet-de-recherche-europeen-BionicAircraft.html

- Carbon 3D (2019) “Our technology“ https://www.carbon3d.com/our-technology/

- Casey T. (2015) "New "waterbone" light weight vehicle design could increase EV battery range" http://cleantechnica.com/2015/04/27/new-waterbone-lightweight-vehicle-design-increase-ev-battery-range/

- Christophe (1985) «Les malices de Plick et Plock» Armand Colin Ed. - Paris.

- Conner B.P., Manogharan G.P., Martof A.N., Rodomsky L.M., Rodomsky C.M., Jordan D.C., Limperos J.W. (2014) "Making sense of 3-D printing: creating a map of additive manufacturing products and services" Additive Manufacturing, 1-4, 64-76.

- Corbel S., André J.C. (1994) « Stéréo-photolithographie laser » Polytechnica Ed.- Paris.

- Cotteleer M.J. (2014) "The 3D opportunity primer - The basics of additive manufacturing" https://dupress.deloitte.com/dup-us-en/focus/3d-opportunity/the-3d-opportunity-primer-the-basics-of-additivemanufacturing.html

- Cotteleer M.J., Trouton S., Dobner E. (2016) “3D opportunity and the digital thread; Additive manufacturing ties it all together" https://dupress.deloitte.com/dup-us-en/focus/3d-opportunity/3d-printing-digital-thread-inmanufacturing.html

- Cunchillos C. (2014) "Les voies de l'émergence - Introduction à la théorie des unités de niveau d'intégration » Belin Ed. - Paris.

- De Gennes P.G., Brochart-Wyart F., Quéré D. (2005) « Gouttes, bulles, perles et ondes » Belin Ed. - Paris.

DGE (2016) « Technologies clés - Préparer l'industrie du futur 2020 http://www.entreprises.gouv.fr/files/files/directions services/politique-et-enjeux/innovation/technologies-cles2020/technologies-cles-2020.pdf

- Delaire J., Piard J., Méallet-Renault R., Clavier G. (2016) « Photo-physique et photochimie ; des fondements aux applications » EDP Sciences Ed. - Paris. 
- Deloitte (2014) « Du nouveau dans le secteur manufacturier : les répercussions de l'impression tridimensionnelle » http://www2.deloitte.com/content/dam/Deloitte/ca/Documents/insights-and-issues/ca-fr-perspectives-et-enjeuxdaffaires-du-nouveau-dans-le-secteur-manufacturier.pdf

- DIRECCTE (2014) " L'impression 3D : Etat des lieux et perspectives » http://www.centre.direccte.gouv.fr/IMG/pdf/L impression 3D etat des lieux et perspectives DIRECCTE Centre en partenariat avec CCI Centre - 10 decembre 2014-2.pdf

- Dodziuk H. (2016) "The Added Value: 3D Printing Brings Much More Than Cheaper Manufacturing" http://3dprintingindustry.com/2016/02/23/the-added-value-3d-printing-carries-much-more-than-cheapermanufacturing/?utm source=3D+Printing+Industry+Update\&utm medium=email\&utm campaign=9a0e5caa42RSS EMAIL CAMPAIGN\&utm term=0 695d5c73dc-9a0e5caa42-64583257

- Ellul J. (1988) «Le bluff technologique » Hachette Ed. - Paris.

- Feynman R. (1959/publication 1960) "There's Plenty of Room at the Bottom" Caltech Engineering and Science, 23, 22-36. http://calteches.library.caltech.edu/47/2/1960Bottom.pdf

- Ford S., Despeisse M. (2016) “Additive manufacturing and sustainability: an exploratory study of the advantages and challenges" Journal of Cleaner Production, 137, 1573-1587.

- France Stratégie (2016) « Quinze ans de politiques d'innovation en France » http://www.strategie.gouv.fr/publications/quinze-ans-de-politiques-dinnovation-france

- Franquin A. (1975) «Spirou et les hommes-bulles/les petits formats » Dupuis Ed. - Marcinelles - Belgique.

- GAO (2015) "3D Printing: opportunities, challenges and policy; implications of additive manufacturing" http://www.gao.gov/assets/680/670960.pdf

- Garetti M., Terzi S., Bertacci N., Brianza M. (2005) "Organisational change and knowledge management in PLM implementation” International Journal of Product Lifecycle Management, $1,43-51$.

- Gleick J. (2008) «La théorie du chaos » Champ Sciences Ed. - Paris.

- Guédon E., Malaquin L., André J.C. (2017) «Bio-printing - État des lieux et perspectives» Techniques de l'Ingénieur, ref. RE268 V1, 23pp.

- Hague R., Reeves P., Jones S. (2016) "Innovate UK - Mapping UK research and innovation in additive manufacturing"

https://www.gov.uk/government/uploads/system/uploads/attachment data/file/496991/CO307 Mapping UK Capabi lity AM WL Print .pdf

- Hart W. (2015) "Faster than Ferrari and it was 3D printed" http://3dprintingindustry.com/2015/11/13/faster-than-aferrari-and-it-was-3dprinted/?utm source $=3 \mathrm{D}+$ Printing + Industry + Update\&utm medium $=$ email\&utm campaign $=06418 \mathrm{cce} 4 \mathrm{f}-$ RSS EMAIL CAMPAIGN\&utm term=0 695d5c73dc-06418cce4f-64557777

- Hunyadi M. (2015) «La tyrannie des modes de vie; sur le paradoxe moral de notre temps » Le bord de l'eau Ed. Lormont.

- Ingelaere R. (2015) «Innovation technologique et performance industrielle : l'exemple de l'impression 3D » http://www.lecese.fr/sites/default/files/pdf/Avis/2015/2015 07 innovations techno impression 3d.pdf

- Irmouli Y., George B., Merlin A. (2009) "Study of the polymerization of acrylic resins by photo-calorimetry: interactions between UV initiators and absorbers" J. Thermal Analysis Calorimetry, 96, 911-916.

- Jessus C. (2017) «Etonnant vivant - Découvertes et promesses du XXIe siècle » CNRS Ed. - Paris.

- Kelly B.E., Bhattacharya I., Heidari H., Shusteff M., Spadaccini C.M., Taylor H.K. (2019) "Volumetric additive manufacturing via tomographic reconstruction" Science, $\underline{363}$, 1075-1079 - DOI: 10.1126/science.aau7114

- Kourilsky F. (2002) «Le chemin de l'interdisciplinarité »17-23 in F. Kourilsky Éd. «Ingénierie de l'interdisciplinarité » L'Harmattan Éd. - Paris.

- Lehn J.M. (2002) “Toward Self-Organization and Complex Matter” Science, 295, 2400-2403.

- Listek V. (2019) "Interview with Nicole Hone who uses 4D Printing to Make Tangible Animation" https://pechealliance.com/la-croissance-massive-de-la-ceramique-fabrication-additive-rapport-de-recherche-dumarche-2019-a-2026/ 
- Loterie D., Delrot P. Moser C. (2019) "High-resolution volumetric additive manufacturing" https://www.researchgate.net/publication/334672136 High-resolution volumetric additive manufacturing

- MADEELI (2017) «Fabrication Additive : Enjeux et Impacts en Occitanie Pyrénées-Méditerranée » http://www.madeeli.fr/wp-content/uploads/2017/01/Fabrication-Additive-en-Occitanie-Etude-2016.pdf

- Mc Ginniss V.D., Schwerzel R.E. (23 Feb. 1984) "Photo-polymerizable composition containing a photosensitizer donor and photo-initiator acceptor" US Patent 4571377 A.

- Melchels F.P.W., Domingos M.A.N., Klein T.J., Maldo J., Bartolo P.J., Hutmacher D.W. (2012) "Additive manufacturing of tissues and organs" Progress in Polym. Sci., 37, 1079-1084.

- Mellor S., Hao L., Zhang D. (2014) “Additive manufacturing: a framework for implementation” Int. J. Prod. Econ., 149, 194-201.

- Mizeret J. (2015) «Les technologies de fabrication additive pour la créativité, le prototypage, la fabrication » http://www.swissmem.ch/fr/presentations-fabrication-additive.html.

- Mirzendehdel A.M., Sureh K. (2016) "Support structure constrained topology optimization for additive

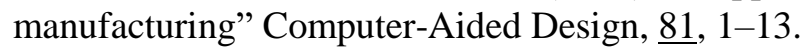

- Mokbel H. (2015) «Vers de nouveaux systèmes amorceurs pour la photopolymérisation radicalaire et/ou cationique dans des conditions plus respectueuses de l'environnement " Thèse Université de Haute Alsace - Mulhouse https://tel.archives-ouvertes.fr/tel-01334572/document

- Moussion A. (2015) «Impression 3D : quel impact sur la propriété intellectuelle ? L’INPI répond dans une interview » http://www.priximprimante3d.com/loi/

- Murr L.E., Johnson W.L. (2017) "3D metal droplet printing development and advanced materials additive manufacturing" Journal of Materials Research and Technology, $\underline{6}, 77-89$.

- OECD (2017) "Key issues for digital transformation in the G20; Report prepared for a joint G20 German Presidency/OECD conference" http://www.oecd.org/G20/key-issues-for-digital-transformation-in-theG20.pdf?utm source=Adestra\&utm medium=email\&utm content=Read\%20the \%20OECD\%27s\%20report\%20for\% 20the $\% 20$ G20\%E2\%80\%A6\&utm campaign=OECD\%20Science\%2C $\% 20$ Technology $\% 20$ and $\% 20$ Innovation $\% 20 \mathrm{Ne}$ ws\%20Jan17\&utm term=demo

- Parrochia D. (1994) «Le statut épistémologique de la vie artificielle»164-185 in F. Tinland «Ordre biologique Ordre technologique » Champ Vallon Ed. - Paris.

- Pictet R. (2015) «Complexité, ordre et hasard en biologie : le cas de l'évolution » 83-96 in J.C. Lévy Ed. « Complexité et désordre ; éléments de réflexion » EDP Sciences Ed. - Paris.

- Pinkerton A.J. (2016) "Lasers in additive manufacturing” Optics \& Laser Tech., 78, 25-32.

- Prigogine I. (1994) “Les lois du chaos » Flammarion Ed. - Paris.

- Rayna T., Striukova L. (2016) "From rapid prototyping to home fabrication: How 3D printing is changing business model innovation" Technological Forecasting and Social Change, 102, 214-224.

- Reeves P. (2012) "Example of Econolyst Research-Understanding the Benefits of AM on CO2" http://www.econolyst.co.uk/resources/documents/files/Presentation 2012 AM and carbon footprint.pdf

- Robinson A. (2015) "The Power of 3D Printing in Manufacturing: An Illustrated Guide" http://advancedmanufacturinginsight.com/archived-articles/item/infographic-3d-printing-manufacturing

- Rufer S. (2014) «Quel est l'impact de l'impression 3d sur la supply chain ?» Mémoire de 1'Université ParisDauphine http://www.memoireonline.com/04/15/9049/Quel-est-l-impact-de-l-impression-3D-sur-la-supply-chain$\underline{. h \mathrm{tml}}$

- Stampfl J., Baudis S., Heller C., Liska R., Neumeister A., Kling R., Ostendorf A., Spitzbart M. (2008) "Photopolymers with tunable mechanical properties processed by laser-based high-resolution stereolithography" J. Micromech. Microeng., 18, 125014.

- Star S.L., Griesemer J.R. (2008) « Ecologie institutionnelle, traductions et objets-frontières : des amateurs et des professionnels au musée de zoologie vertébrée de Berkeley 1907-1939 » in B. Lahire et C. Rosental Ed. « La cognition au prisme des sciences sociales » Editions des Archives Contemporaines - Paris.

- Shusteff M., Browar A.E.M., Kelly B.E., Henriksson J., Weisgraber T.H., Panas R.M., Fang N.X., Spadaccini C.M. (2017) "One-step volumetric additive manufacturing of complex polymer structures" Science Advances, $\underline{3}$, eaao5496 - DOI: 10.1126/sciadv.aao5496 
- Tang Y., Mak K., Zhao Y.F. (2016) "A framework to reduce product environmental impact through design optimization for additive manufacturing” Journal of Cleaner Production, 137, 1560-1572.

- Tarit J.P. (2018) «Le futur des pièces de rechange est en 3D » https://ethikis.com/reparabilite-et-impression-3d-jptarrit/

- Töpffer R. (1835) cité par Bouveresse J. (2017) «Le mythe moderne du progrès » Agone Ed. - Marseille.

- Vaezi M., Seitz H., Yang S. (2013) “A review on 3D micro-additive manufacturing technologies” Int. J. Adv. Manuf. Technol., $\underline{67}, 1721-1754$.

- Valero J. (2016) "Industry 4.0: The future is here" http://www.euractiv.com/section/innovationindustry/linksdossier/industry-4-0-the-future-of-manufacturing-is-here/\#ea-accordion-further-reading

- Vandeginste P. (2012) « Modéliser le vivant » http://www.larecherche.fr/savoirs/dossier/4-modeliser-vivant-01-052012-91189

- Verhulst J. (2015) “Analyse de l'état et de l'avenir du marché de l'impression 3d” Master en sciences de gestion de l'Université Catholique de Louvain $\quad$ B http://dial.uclouvain.be/memoire/ucl/en/object/thesis\%3A2710/datastream/PDF 01/view

- Viveret P. (2012) «La cause humaine; du bon usage de la fin du monde » Les liens qui libèrent Ed. - Paris.

- Wang P., Chu W., Li W., Tan Y., Liu F., Wang M., Wang Z., Qi J., Lin J., Zhang F., Wang Z., Cheng Y. (2019) "Three-dimensional laser printing of macroscale glass objects at a micro-scale resolution" https://arxiv.org/ftp/arxiv/papers/1904/1904.04027.pdf

- Weller C., Kleer R., Piller F. (2015) "Economic implications of 3D printing: Market structure models in light of additive manufacturing revisited" Int. J. Production Economics, 164, 43-56.

- Zarek M., Mansour N., Shapira S., Cohn D. (2017) “4D Printing of Shape Memory-Based Personalized Endoluminal Medical Devices” Macromol. Rapid Commun. 1600628. DOI: 10.1002/marc.201600628. 